\title{
A study of the flow-field evolution and mixing in a planar turbulent jet using direct numerical simulation
}

\author{
By S. A. STANLEY†, S. SARKAR AND J. P. MELLADO \\ University of California, San Diego, La Jolla, CA 92093-0411, USA
}

(Received 26 March 1999 and in revised form 23 July 2001)

Turbulent plane jets are prototypical free shear flows of practical interest in propulsion, combustion and environmental flows. While considerable experimental research has been performed on planar jets, very few computational studies exist. To the authors' knowledge, this is the first computational study of spatially evolving three-dimensional planar turbulent jets utilizing direct numerical simulation. Jet growth rates as well as the mean velocity, mean scalar and Reynolds stress profiles compare well with experimental data. Coherency spectra, vorticity visualization and autospectra are obtained to identify inferred structures. The development of the initial shear layer instability, as well as the evolution into the jet column mode downstream is captured well.

The large- and small-scale anisotropies in the jet are discussed in detail. It is shown that, while the large scales in the flow field adjust slowly to variations in the local mean velocity gradients, the small scales adjust rapidly. Near the centreline of the jet, the small scales of turbulence are more isotropic. The mixing process is studied through analysis of the probability density functions of a passive scalar. Immediately after the rollup of vortical structures in the shear layers, the mixing process is dominated by large-scale engulfing of fluid. However, small-scale mixing dominates further downstream in the turbulent core of the self-similar region of the jet and a change from non-marching to marching PDFs is observed. Near the jet edges, the effects of large-scale engulfing of coflow fluid continue to influence the PDFs and non-marching type behaviour is observed.

\section{Introduction}

Turbulent plane jets are prototypical free shear flows on which fundamental research can expand the overall understanding of turbulent flows. In addition, turbulent plane jets are of practical interest due to their presence in a broad range of engineering applications such as combustion, propulsion, and environmental flows. A thorough understanding of the dynamics of these flows is required in order to understand and control the transport processes in these applications.

There have been many experimental studies of planar turbulent jets over the years. Most of the early work concentrated on quantifying the statistical properties of the self-similar region far downstream. Albertson et al. (1950) and Miller \&

$\dagger$ Current address: Lawrence Berkeley National Laboratory, 50A-1148, Berkeley, CA 94720, USA. 
Comings (1957) measured the mean profiles of the velocity and pressure fields in the jet. Utilizing hot-wire anemometry, Bradbury (1965), Heskestad (1965) as well as Gutmark \& Wygnanski (1976) were later able to measure the mean and fluctuating fields in the self-similar region of planar jets. They found that the evolution of the fluctuating velocity fields as well as the jet spreading rate and centreline velocity decay were strongly affected by the initial conditions at the nozzle and the external conditions in the laboratory. In addition, Everitt \& Robins (1978), Bradbury \& Riley (1967) as well as recent work of LaRue et al. (1997) showed that the presence of a strong coflow can slow the development of the jet to a self-similar state.

While there has been a large amount of experimental work performed on planar jets, the number of numerical studies is small. Comte et al. (1989) performed temporal simulations of the fully developed region of a two-dimensional Bickley jet to study the influence of initial conditions. Reichert \& Biringen (1997) studied the effects of compressibility on the spatial evolution of two-dimensional, inviscid jets with a strong coflow. Stanley \& Sarkar (1997a,b) compared two-dimensional weak and strong jets with three-dimensional jets and found that two-dimensional simulations of strong planar jets lead to incorrect mean velocity profiles and are therefore of little use in the study of real jets.

Dai, Kobayashi \& Taniguchi (1994) performed the first simulations of a threedimensional spatially evolving, subsonic, plane jet using large-eddy simulation (LES). They obtained relatively good agreement with experimental data in their mean profiles; however the self-similar turbulence intensities were higher by approximately $40 \%$. More recently, Weinberger, Rewerts \& Janicka (1997) showed self-similar fluctuation intensities which were $15 \%$ higher than nominal values in their large-eddy simulation using the Smagorinsky model. However, neither of these studies analysed the influence of the LES model. Le Ribault, Sarkar \& Stanley (1999) performed an extensive comparison of the Smagorinsky, dynamic Smagorinsky and dynamic mixed LES models in simulations of the near-field region of planar turbulent jets and found that the latter two models resulted in good agreement both with direct numerical simulation (DNS) and higher Reynolds number laboratory experiments.

There is great interest in the transport and mixing processes of scalars in turbulent shear flows due to their importance in the propagation of contaminants in environmental flows, as well as the wide range of applications involving turbulent combustion. The evolution of the probability density function (PDF) of a passive scalar has been utilized to study the mixing process in planar turbulent shear layers (Batt 1977; Koochesfahani \& Dimotakis 1986; Rogers \& Moser 1994; Karasso \& Mungal 1996), as well as in round jets, for example, Dahm \& Dimotakis 1990. However, there remains considerable debate on the final downstream state of the mixing process in self-similar shear layers.

The intention of this work is twofold. First, to develop an accurate computational model for a spatially evolving turbulent plane jet that has been rigorously validated against experimental data. Then, to utilize this model to provide thorough documentation of a particular planar jet which can be utilized in the design and evaluation of models for large-eddy simulation. Not only are mean velocity and turbulence intensity profiles provided, but also the development of shear layer structures into jet flow structures is documented by vorticity visualization and coherence spectra. It is recognized that the inflow conditions can have a long-lived downstream effect on the development of turbulent flowfields. George (1989) suggested a coupling between the initial conditions in a flow field and the resulting self-similar state through the evolution of large-scale coherent structures. Likewise, Rogers \& Moser (1994) as well 
as Moser, Rogers \& Ewing (1998) observed an influence of the initial conditions in direct numerical simulation of a temporally evolving mixing layer on the final self-preserving state. This coupling, be it strong or weak, is present in experimental as well as numerical studies of turbulent flows. By providing thorough documentation of a planar jet for which the initial conditions are well defined and reproducible, this simulation serves as a baseline for the evaluation of turbulence models. This numerical model has already been utilized (Le Ribault et al. 1999, 2001) for the evaluation of several existing 'standard' subgrid-scale models for large-eddy simulation.

Secondly, this computational model has been utilized to study two physical properties of turbulent planar jets which have not been previously documented: the small-scale anisotropy and the evolution of the mixing properties of the jet. While the large-scale anisotropy has been well documented through experimental studies, the relative level of isotropy of the small scales of the turbulent motion in planar jets has been less studied. In general, evaluating the spatial derivatives in all three directions of the three components of the fluctuating velocity field is difficult to do experimentally. Similarly, while mixing has been studied in round jets as well as shear layers, the mixing process in planar jets has not been documented.

To the authors' knowledge, this work represents the first study of spatially evolving turbulent plane jets utilizing DNS. The DNS approach has been utilized in the study of free turbulent shear flows which are homogeneous in one or more directions, that is, temporal simulations. However, relatively few direct numerical simulations of spatially evolving turbulent flows have been performed.

\section{Governing equations}

The governing equations applicable in the study of compressible, turbulent shear flows consist of the conservation equations for mass, momentum and energy. In this study we assume an ideal gas with a Newtonian relationship between fluid stress and rate of strain. The non-dimensional conservation equations are summarized as follows:

mass conservation

$$
\frac{\partial \rho}{\partial t}+\frac{\partial\left(\rho u_{k}\right)}{\partial x_{k}}=0
$$

momentum conservation

$$
\frac{\partial\left(\rho u_{i}\right)}{\partial t}+\frac{\partial\left(\rho u_{i} u_{k}\right)}{\partial x_{k}}=-\frac{\partial p}{\partial x_{i}}+\frac{1}{R e} \frac{\partial \tau_{i j}}{\partial x_{j}},
$$

where the viscous stress tensor, $\tau_{i j}$, is given by

$$
\tau_{i j}=\left(\frac{\partial u_{i}}{\partial x_{j}}+\frac{\partial u_{j}}{\partial x_{i}}\right)-\frac{2}{3} \frac{\partial u_{k}}{\partial x_{k}} \delta_{i j} ;
$$

energy conservation

$$
\frac{\partial p}{\partial t}+u_{k} \frac{\partial p}{\partial x_{k}}=-\gamma p \frac{\partial u_{k}}{\partial x_{k}}+\frac{\gamma}{\operatorname{Pr} R e} \frac{\partial^{2} T}{\partial x_{k}^{2}}+\frac{\gamma-1}{\operatorname{Re}} \Phi,
$$

where $\Phi$ is the viscous dissipation function,

$$
\Phi=\tau_{i j} \frac{\partial u_{i}}{\partial x_{j}}
$$


The values of the fluid thermodynamic quantities are related through the equation of state for an ideal gas,

$$
p=\rho R T \text {. }
$$

Additionally, a scalar equation is included which expresses the conservation of a passive property, $\xi$ :

$$
\frac{\partial(\rho \xi)}{\partial t}+\frac{\partial\left(\rho u_{k} \xi\right)}{\partial x_{k}}=\frac{1}{\operatorname{ReSc}} \frac{\partial}{\partial x_{k}}\left(\rho \frac{\partial \xi}{\partial x_{k}}\right) .
$$

The property $\xi$ is passive in that it is influenced by the flow without itself having any influence on the flow. This type of scalar equation is commonly used to study mixing in turbulent shear flows and is applicable to chemical systems under the assumption of infinitely fast chemistry, a useful simplification (Williams 1985) in combustion studies.

\section{Numerical techniques}

The techniques used in this study to numerically solve the governing equations $\S 2$, are summarized here. Further details may be found in Stanley \& Sarkar (2000). The general requirements for any study of turbulence using direct numerical simulation is that the numerical techniques provide high accuracy in both space and time as well as be computationally efficient. During the development of the numerical algorithm described below a balance between accuracy and efficiency was sought.

The Euler terms in the governing equations discussed in $\S 2$ are marched in time using the low-storage, fourth-order Runge-Kutta integration scheme of Carpenter \& Kennedy (1994). The viscous and conduction terms are marched in time using a first-order integration scheme leading to a $20 \%$ reduction in computational time without significant loss of accuracy. This is implemented by advancing the Euler terms in time using the Runge-Kutta scheme described above and then evaluating and advancing the viscous terms using a first-order scheme.

A non-uniform sixth-order compact derivative scheme based on the uniform scheme of Lele (1992) is utilized to evaluating the spatial derivatives. This central-derivative scheme is closed at the boundaries using internal-biased, non-uniform, third-order compact derivatives based on the uniform derivatives of Carpenter, Gottlieb \& Abarbanel (1993). This 3-6-3 scheme allows the simulation of problems on an open, non-periodic domain while maintaining at least an overall fourth-order spatial accuracy in the physical coordinate $\Delta x$ of the non-uniform grid. In order to eliminate high-wavenumber errors resulting from numerical boundary closures, a non-uniform fourth-order compact filter is utilized to damp the high-wavenumber modes. As with the compact derivatives, this non-uniform compact filter generalizes the uniform filter of Lele (1992) to non-uniform meshes providing fourth-order accuracy in $\Delta x$. Only modes between the highest wavenumber, of frequency $f_{x} \Delta x=0.5$, and $f_{x} \Delta x=0.43$ are significantly affected by the filter. The jet Reynolds number and grid resolution are chosen such that the dissipation due to filtering is less than $1 \%$ of the viscous dissipation.

At the subsonic inflow boundary, the governing equations are essentially solved in a characteristic form. The time variations of the incoming characteristic variables are specified while the equation for the outgoing characteristic variable is solved using internal biased derivatives. Through the solution of the equation for the outgoing acoustics, the actual values of the variables at the inflow plane are allowed to 
float around the specified 'target' values thus avoiding sharp changes during the downstream evolution as well as avoiding the introduction of excessive dilatation, $\nabla \cdot \boldsymbol{u}$, into the flow.

For the downstream boundary and the two sidewall boundaries, the non-reflecting boundary conditions of Thompson $(1987,1990)$ are used. The form of these conditions is allowed to switch between that for non-reflecting inflow and outflow at each point on the boundary depending on the instantaneous local normal velocity. The two corner points on the outflow boundary are treated as non-reflecting at an angle $45^{\circ}$ from the two adjacent boundaries, and the two corner points on the inflow boundary are calculated using the characteristic inflow conditions. At all outflow points on the downstream and sidewall boundaries, the pressure correction term originally proposed by Rudy \& Strikwerda (1980) and later discussed by Poinsot \& Lele (1992) is utilized.

It should be noted that these characteristic-based local boundary conditions were derived based on linearized waves propagating normal to the boundary and, thus, are not strictly non-reflecting in a multi-dimensional nonlinear flow. When the direction of wave propagation at the boundaries deviates from normal incidence, the amount of spurious reflection increases, necessitating the use of filters for long-time stability of the turbulent jet simulations. In addition, a perfectly matched layer (PML) buffer zone based on that of $\mathrm{Hu}$ (1996) was utilized on the downstream and sidewall boundaries in order to further isolate the interior of the domain from the effects of the boundary conditions. In Stanley \& Sarkar $(1997 b)$ it was demonstrated that this boundary condition scheme is effective at allowing large-scale structures to pass out of the computational domain through the outflow boundary. The influence of the outflow is limited to the region of the domain very near this boundary. Since there is a possible influence of the boundary on the region of the domain near the outflow, data from this region will not be utilized in the statistical analysis of the jet.

The computational grid used in this study was generated using a simple geometric progression, $\Delta y_{j+1}=A_{j} \Delta y_{j}$. Further details are provided later regarding the specific grid used in each of the simulations.

\section{Inflow conditions}

The longitudinal velocity in the shear layer on either side of the jet at the inflow is given by a hyperbolic tangent profile,

$$
u=\frac{U_{1}+U_{2}}{2}+\frac{\Delta U_{o}}{2} \tanh \left(\frac{y}{2 \theta_{o}}\right),
$$

where $\theta_{o}$ is the shear layer momentum thickness, $U_{1}$ and $U_{2}$ are the velocities of the jet and the coflow respectively and $\Delta U_{o}=U_{1}-U_{2}$. The subscript on $\Delta U$ and $\theta$ indicates inflow values. This profile is mirrored about the jet centreline to obtain a top-hat mean jet profile with smooth edges. The mean lateral and spanwise velocities are zero at the inflow. The mean pressure and density at the inflow are initially uniform, although a slight variation across the jet is generated due to the outgoing acoustic waves.

The mean profile for the passive scalar at the inflow is given by a hyperbolic tangent profile

$$
\xi=\frac{\xi_{1}+\xi_{2}}{2}+\frac{\xi_{1}-\xi_{2}}{2} \tanh \left(\frac{y}{2 \theta_{o}}\right)
$$



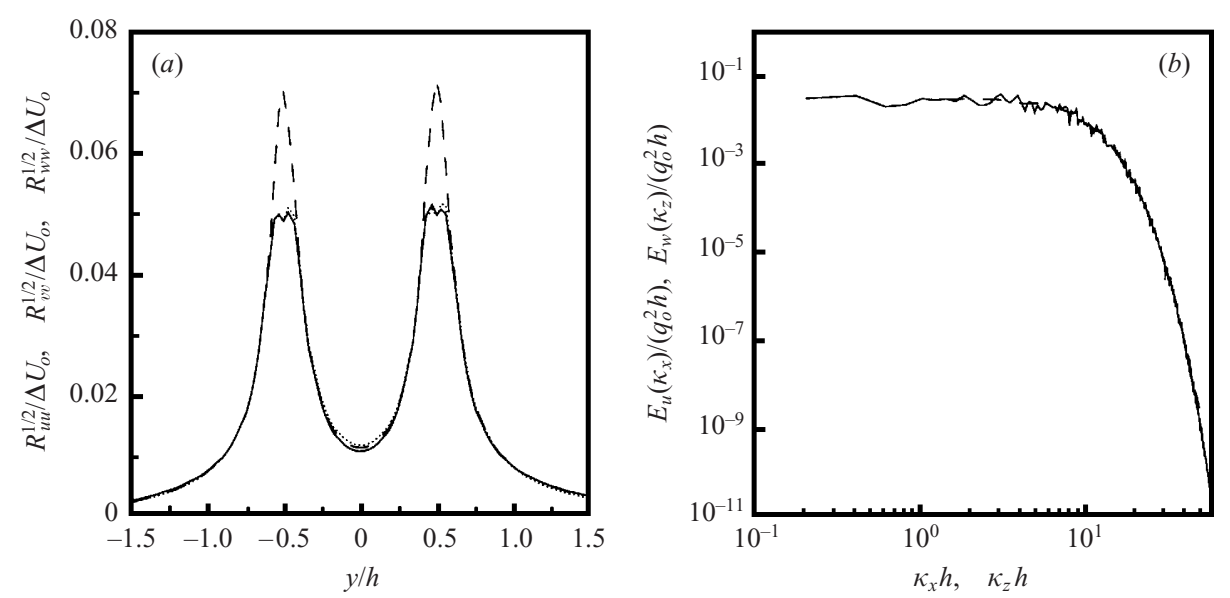

FIGURE 1. Reynolds stress profiles and one-dimensional autospectra of the broadband fields utilized to force the three-dimensional jet simulations. (a) Reynolds stress profiles.,$- R_{u u} ;---, R_{v v}$; $\cdots \cdots, R_{w w}$. (b) One-dimensional autospectra.,$- E_{u}\left(\kappa_{x}\right) ;---, E_{w}\left(\kappa_{z}\right)$.

with $\xi_{1}=1.0$ and $\xi_{2}=0$ in the jet and ambient, respectively. The value of $\theta_{o}$ used for the passive scalar is the same as that used for the mean longitudinal velocity profile.

A broadband inflow forcing is utilized with a three-dimensional energy spectrum specified by

$$
E(\kappa)=\frac{4 K}{\kappa_{o}}\left(\frac{\kappa}{\kappa_{o}}\right)^{2} \exp \left(-2 \frac{\kappa}{\kappa_{o}}\right),
$$

$K$ being the turbulent kinetic energy. The peak wavenumber, $\kappa_{o}$, is specified to be the fundamental mode for the hyperbolic tangent shear layer given by $f_{o} \theta_{o} / U_{c}=0.033$ (Michalke 1965; Monkewitz \& Huerre 1982) where $U_{c}=\left(U_{1}+U_{2}\right) / 2$ is the convection velocity and $f_{o}$ is the temporal frequency $\left(\kappa_{o}=2 \pi f_{o} / U_{c}\right)$. This forcing is designed to provide energy to the flow field in a range of scales characteristic of that present in an actual turbulent flow in order to increase the rate at which the jet develops from the top-hat profile present at the inflow plane to the self-similar profiles downstream. This broadband forcing of the inflow plane is performed by generating a three-dimensional volume containing fluctuating solenoidal velocity, pressure and density fields which is then convected past the inflow plane, as the simulation is integrated in time, using a constant convection velocity, $U_{c}$. Figure $1(a)$ shows the profiles of the Favre-averaged Reynolds stresses, $R_{i j}=\widehat{u_{i}^{\prime \prime} u_{j}^{\prime \prime}}=\overline{\rho u_{i}^{\prime \prime} u_{j}^{\prime \prime}} / \bar{\rho}$, for the broadband fields used to force the inflow plane in the simulation discussed in $\S 5$. The strong peaks in the shear layers on either side of the jet are clear, as well as the strong drop in intensity towards the jet centreline. While there are fluctuations present in the pressure and density at the inflow, their magnitude is small. The root-mean-square pressure fluctuations have a peak intensity of $p_{r m s}^{\prime} / \bar{\rho} \Delta U_{o}^{2}=2.7 \times 10^{-2}$ while the peak in the density fluctuations is $\rho_{r m s}^{\prime} / \bar{\rho}=2.8 \times 10^{-3}$. Figure $1(b)$ shows the one-dimensional autospectra in the $x$ - and $z$-directions of the longitudinal and spanwise components of velocity, respectively, inside the lower shear layer. The autospectra are defined to yield

$$
R_{u u}=\int_{0}^{\infty} E_{u}\left(\kappa_{x}\right) \mathrm{d} \kappa_{x}
$$


and similarly for $R_{w w}$. The energy contained in these fluctuating fields is spread over a broad spectrum in both time (streamwise) and the spanwise direction.

\section{Evolution of the velocity field}

This section discusses the evolution of a spatially evolving jet with Reynolds number $R e_{h}=\bar{\rho} \Delta U_{o} h / \mu=3000$ and turbulent inflow. Here $h$ is the jet nozzle width and $\Delta U_{o}$ is the difference between the centreline velocity and the coflow velocity. The Reynolds number based on the jet width increases to approximately 4800 at the outflow. The Taylor microscale Reynolds number, $R e_{\lambda}=\bar{\rho} \lambda q / \mu$, on the jet centreline at the outflow is 143, where $\lambda^{2}=5 \mu q^{2} /(\bar{\rho} \epsilon)$ is the three-dimensional Taylor microscale and $q^{2}=$ $\overline{u^{\prime} u^{\prime}}+\overline{v^{\prime} v^{\prime}}+\overline{w^{\prime} w^{\prime}}$. The Schmidt number, $S c=\mu / \bar{\rho} D_{\xi}$, and Prandtl number, $\operatorname{Pr}=C_{p} \mu / k$, are 1.0 and 0.72 , respectively, for this simulation. The mean jet velocity at the inflow is given by equation (4.1) with a velocity ratio, $\eta=\Delta U_{o} /\left(U_{1}+U_{2}\right)=0.83$. With this velocity ratio the coflow velocity in this jet is small compared to the velocity difference, $U_{2} / \Delta U_{o}=0.1$. Although this simulation is performed using the compressible NavierStokes equations, the convective Mach number, $M_{c}=\Delta U_{o} /\left(c_{1}+c_{2}\right)=0.16$, is such that the flow field is essentially incompressible. This is demonstrated by the fact that the density fluctuation is small, going through a local peak of $\overline{\rho^{\prime 2}} / \bar{\rho}^{2}=5.9 \times 10^{-5}$ in the shear layers at $x / h=6.4$ and approaching $\overline{\rho^{\prime 2}} / \bar{\rho}^{2}=1.8 \times 10^{-5}$ at $x / h=12.0$. Down the centreline of the jet the maximum density fluctuation intensity is $\overline{\rho^{\prime 2}} / \bar{\rho}^{2}=2.7 \times 10^{-5}$. The momentum thickness of the shear layers at the inflow is $\theta_{o} / h=0.05$. The inflow forcing for this jet has the spectrum given by equation (4.3), while its cross-stream profile is set to have a maximum intensity of $q_{o} / \Delta U_{o}=0.10$ in the shear layers, decaying down to $q_{o} / \Delta U_{o}=1.9 \times 10^{-2}$ at the centreline of the jet. This simulation was performed on a $390 \times 390 \times 130$ computational grid with a physical domain size of $L_{x} / h=13.5+1.5, L_{y} / h=13.0+3.0, L_{z} / h=4.3$ and a grid spacing in the domain interior of $\Delta x=\Delta y=\Delta z=0.033 \mathrm{~h}$. At the outflow of the computational domain, the grid spacing relative to the Kolmogorov scale, $\eta=\mu^{3 / 4} /\left(\epsilon \bar{\rho}^{3}\right)^{1 / 4}$, is $\Delta x / \eta=3.2$, while the maximum value is $\Delta x / \eta=3.4$, at $x / h=7.9$. The designation, $L / h=a+b$, for the domain dimensions indicates that the interior of the domain has size $a$ while there is a buffer zone of size $b$. The simulation is performed for 3 flow time units, $t\left(U_{1}+U_{2}\right) / 2 L_{x}=3$, to obtain converged statistics. Such a time is required so as to average over the flapping of the jet. The CPU time is 21665 Cray T3E hours.

In the course of the discussion below, comparisons of the current results against experimental data are made. The studies of Thomas \& Chu (1989); Thomas \& Prakash (1991) and Browne et al. (1983) are used for comparison of the downstream evolution of the jet width, centreline velocity decay, and centreline fluctuation intensities because they document the near field of the jet well. However, these studies do not provide complete measurements of the fully developed region of the jet. The data in the fully developed region of Gutmark \& Wygnanski (1976) and Ramaprian \& Chandrasekhara (1985) are used for evaluating the self-similar behaviour. The physical conditions for these experimental studies are outlined in table 1 for comparison with the physical conditions of the current study. Note that, despite the relatively short streamwise domain length in the DNS, the mean and turbulence profiles near the outflow approach the expected self-similar values because of the turbulent inflow conditions.

The fundamental mode observed experimentally in the fully developed region of planar jets is $S=f_{j t}^{*} \delta_{U} / \Delta U_{c}=0.11$ where $\delta_{U}$ is the jet half-width and $\Delta U_{c}$ is the 


\begin{tabular}{lcccc} 
Source & $R e_{h}$ & $h / \theta$ & {$[q / \Delta U]_{s l}$} & {$[q / \Delta U]_{c l}$} \\
DNS results & 3000 & 20 & 0.100 & 0.019 \\
Ramaprian \& Chandrasekhara (1985) & 1600 & $29 \dagger$ & - & - \\
Browne et al. (1983) & 7620 & 54 & - & 0.0035 \\
Thomas \& Prakash (1991) & 8000 & 67 & - & $\leqslant 0.0042$ \\
Thomas \& Chu (1989) & 8300 & 67 & - & $\leqslant 0.0043$ \\
Gutmark \& Wygnanski (1976) & 30000 & - & - & 0.0035 \\
Hussain \& Clark (1997) & 32550 & 182 & 0.071 & 0.021 \\
\multicolumn{5}{|}{$\dagger$ Momentum thickness, $\theta$, estimated based on laminar flow in the nozzle. } \\
The DNS parameters are also given.
\end{tabular}

TABLE 1. Physical parameters of the experimental results used for comparison.

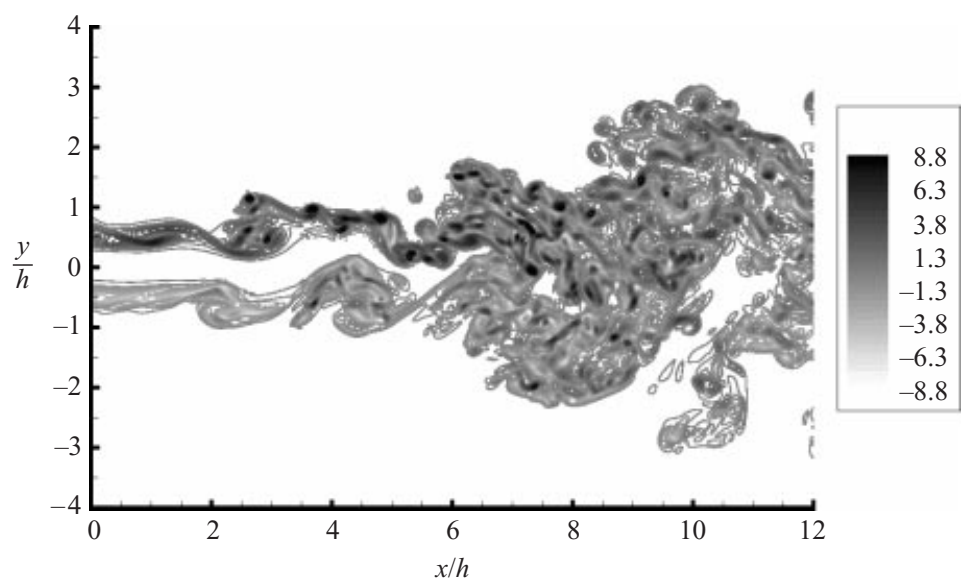

FIGURE 2. Instantaneous spanwise vorticity, $\omega_{z}$, contours on an $(x, y)$-plane located at $z / h=2.15$.

velocity difference between the local centreline value and the local coflow velocity. In terms of the jet scaling, the fundamental mode for the shear layers at the inflow of this jet is $f_{s l}^{*} \delta_{U} / \Delta U_{c}=0.197$ and is therefore not a harmonic of the jet mode.

\subsection{Structural development of the jet}

Figure 2 shows instantaneous contours of the spanwise vorticity, $\omega_{z}$, on an $(x, y)$-plane through the domain at $z / h=2.15$. In the region $0 \leqslant x / h \leqslant 4.0$ there is clear indication of the presence of vortex rollup in both the upper and lower shear layers. In the lower shear layer, peaks in the magnitude of the spanwise vorticity are present at $x / h=4.0$ and $x / h=2.0$. The streamwise wavelength of the spanwise vortices is consistent with the expected wavelength of the shear layer instability of $\lambda_{s l}^{*} / h=\left(U_{c} / f_{s l}^{*}\right) / h=1.52$, taking into account that the broadening of the shear layers increases this value. A large engulfing of outer irrotational fluid is present downstream, and the same process is observed in the middle of the domain, involving this time the potential core of the jet immediately after the nozzle. This mechanism is responsible for the presence of irrotational parcels of fluid inside the turbulent region, as shown by figure 4 and figure 5 .

Figure 3 shows the coherency spectra of the lateral velocity at two points in the shear layers on opposite sides of the jet, $y / \delta_{U}= \pm 1.0$. It is calculated by the Fourier 


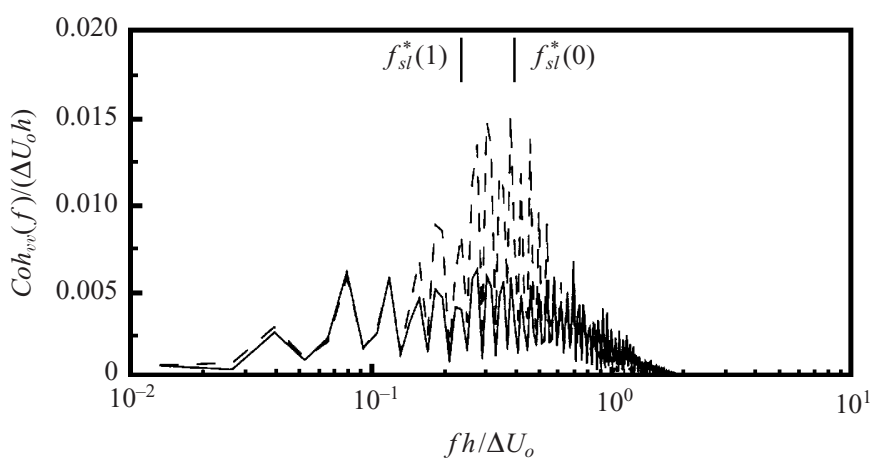

FIGURE 3. Two-point lateral velocity coherence spectra for points in the upper and lower shear layers, $y / \delta_{U}= \pm 1.0$, at the stations $x / h=0(-)$ and $x / h=1.0(---)$.

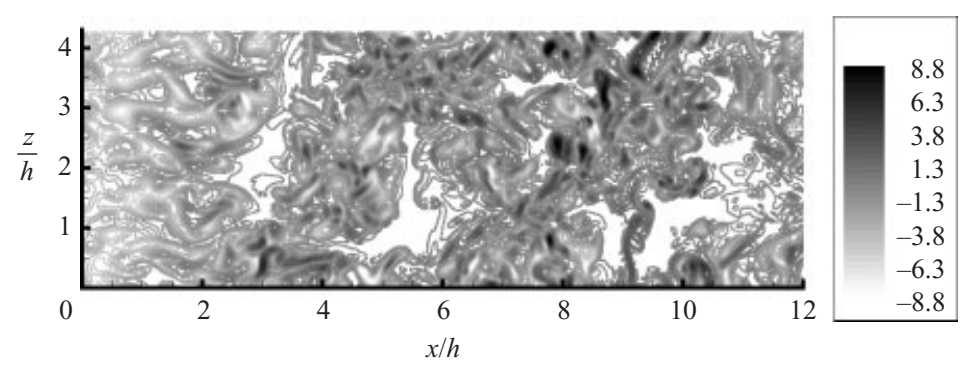

FIGURE 4. Instantaneous spanwise vorticity, $\omega_{z}$, contours on an $(x, z)$-plane in the lower shear layer, $y / h=-0.50$.

transform

$$
\operatorname{Coh}_{v v}(f)=\left|E_{v v}(f)\right|=\left|\int_{-\infty}^{\infty} C_{v v}(\tau) \mathrm{e}^{-2 \pi \mathrm{i} f \tau} \mathrm{d} \tau\right|,
$$

where $C_{v v}$ is the two-time correlation $\overline{v_{A}^{\prime}(t) v_{B}^{\prime}(t+\tau)}$ corresponding to two spatial points $\mathrm{A}$ and $\mathrm{B}$. The use of the coherency spectra reduces the influence of the broadband background energy and accentuates the discrete frequencies which are present on each side of the jet. Also shown in the figure is the most unstable frequency for the shear layer based on the scaling at $x / h=0, f_{s l}^{*}(0)$, as well as based on the scaling at $x / h=1.0, f_{s l}^{*}(1)$. The most unstable frequency is defined using the fundamental mode for the hyperbolic tangent shear layer, $S=f_{s l}^{*} \theta / U_{c}=0.033$, so that $f_{s l}^{*}(x)=0.033 U_{c}(x) / \theta(x)$. It is clear from the coherency spectra that in this region, the strongest growing mode is at a frequency between $f_{s l}^{*}(0)$ and $f_{s l}^{*}(1)$. It seems likely that the strong growth of this mode is a result of the shear layer instability. However, the spreading of the shear layer in this region results in this mode appearing at a frequency in between $f_{s l}^{*}(0)$ and $f_{s l}^{*}(1)$. There is also a strong peak which almost coincides with the most unstable mode for the shear layer at the inflow, $f_{s l}^{*}(0)$.

Figure 4 shows an instantaneous $(x, z)$-plane of the spanwise vorticity through the lower shear layer, $y / h=-0.50$. While there is some spanwise variation of $\omega_{z}$ in the shear layer for $0 \leqslant x / h \leqslant 2.0$, in general the structures appear to be relatively twodimensional. The spanwise variations present in this region are small and of relatively long wavelength. However, downstream of $x / h=2.0$ there is a rapid increase in the three-dimensionality of the structures present in the shear layer. This breakdown of the spanwise Kelvin-Helmholtz rollers coincides with a strong growth in the magnitude 


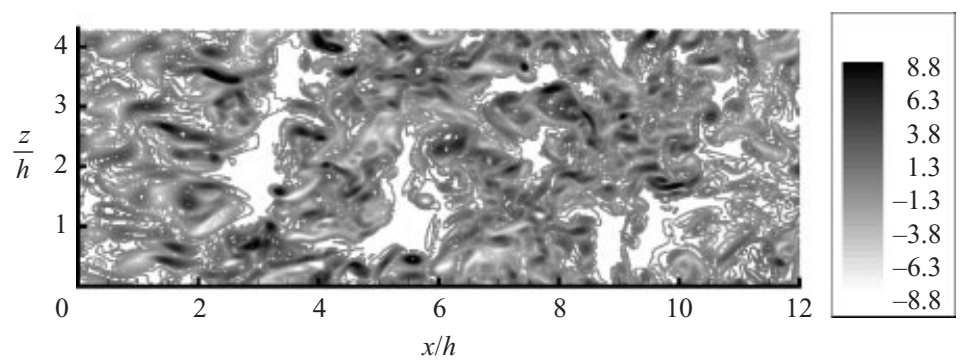

FIGURE 5. Instantaneous streamwise vorticity, $\omega_{x}$, contours on an $(x, y)$-plane in the lower shear layer, $y / h=-0.50$.

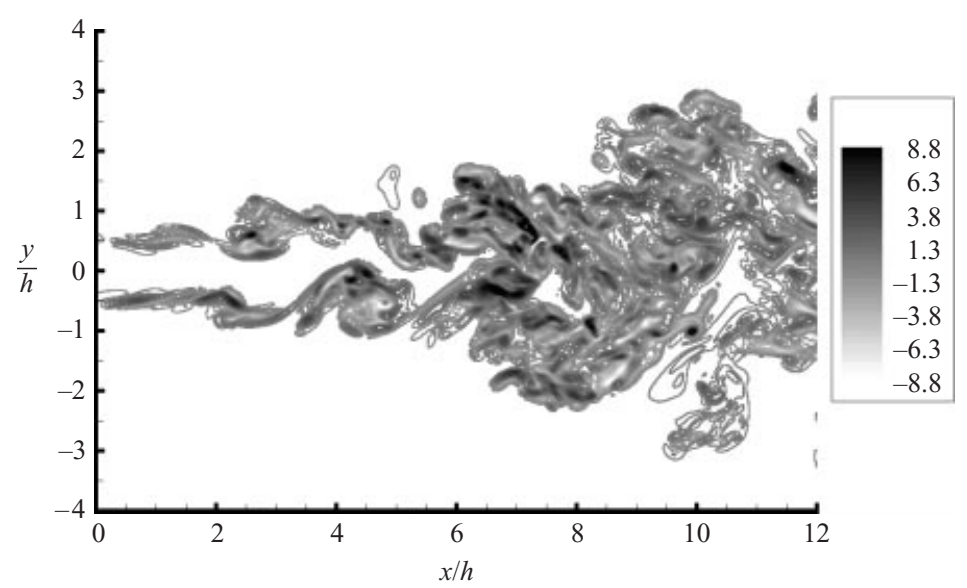

FIGURE 6. Instantaneous streamwise vorticity, $\omega_{x}$, contours on an $(x, y)$-plane, $z / h=2.15$.

of the streamwise vorticity, figure 5. While there is streamwise vorticity present in the shear layers at the inflow, only downstream of $x / h=2.0$ is the magnitude of the streamwise vorticity comparable with that of the spanwise vorticity.

Figure 6 shows an $(x, y)$-plane of the streamwise vorticity, $\omega_{x}$, at the spanwise station, $z / h=2.15$. From figures 5 and 6 it is clear that the structures present in the streamwise vorticity for $0 \leqslant x / h \leqslant 4.0$ are elongated in the streamwise direction and have an inclination inwards towards the centreline of the jet suggesting stretching by the mean strain of the jet. The behaviour of the structures in the spanwise and streamwise vorticity for $0 \leqslant x / h \leqslant 4.0$ is consistent with the rollup and pairing of spanwise vortices, generation of streamwise vortices, and eventual breakdown to strong three-dimensional turbulence which is observed in shear layers (for example Lasheras \& Choi 1988). It is clear that the beginning of the breakdown to strongly three-dimensional turbulence occurs before the merging of the shear layers for the jet simulated here.

The strong vorticity patches in the two shear layers near the inflow show signs of interacting in the region $4.0 \leqslant x / h \leqslant 6.0$. A few small patches of streamwise and spanwise vorticity begin to appear near the jet centreline in this range, figures 2 and 6. However, only downstream of $x / h=6.0$ are there structures present in the vorticity field near the centreline of a magnitude similar to that present in the highshear region. In the downstream region of the domain, $x / h \geqslant 8.0$, the distribution of vorticity across the jet is relatively uniform from the upper shear region across the 


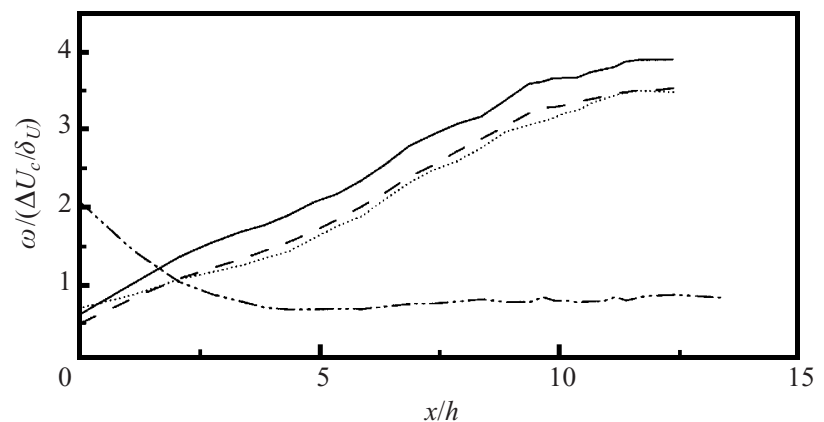

FIGURE 7. Downstream growth of the root-mean-square vorticity and mean spanwise vorticity in the lower high-shear region, $\left.\left.y / \delta_{U} \approx-1.0 .-, \overline{\left(\omega_{x}^{\prime 2}\right.}\right)^{1 / 2} /\left(\Delta U_{c} / \delta_{U}\right) ;---, \overline{\left(\omega_{y}^{\prime 2}\right.}\right)^{1 / 2} /\left(\Delta U_{c} / \delta_{U}\right) ; \cdots \cdots$, $\left(\overline{\omega_{z}^{\prime 2}}\right)^{1 / 2} /\left(\Delta U_{c} / \delta_{U}\right) ;-\cdot-, \overline{\omega_{z}} /\left(\Delta U_{c} / \delta_{U}\right)$.

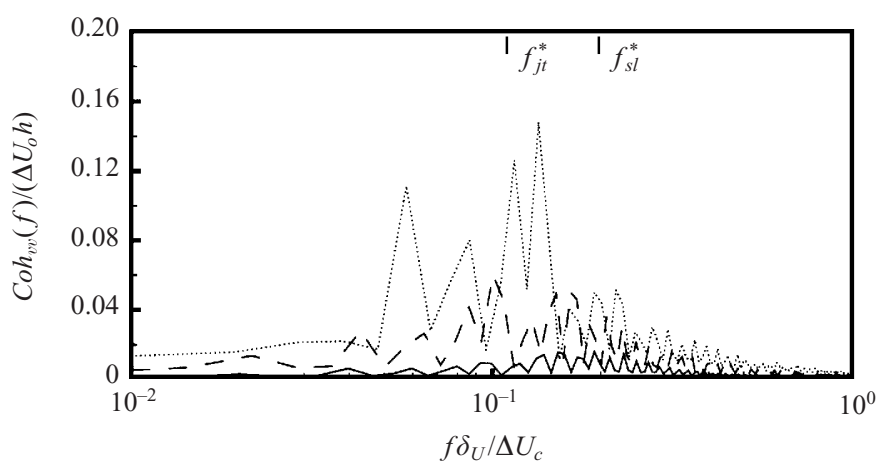

FIGURE 8. Two-point lateral velocity coherence spectra for points in the upper and lower shear layers, $y / \delta_{U}= \pm 1.0$, for $1.0 \leqslant x / h \leqslant 5.0$. $-x / h=1.0 ;---, x / h=3.0 ; \cdots \cdots, x / h=5.0$.

centreline to the lower shear region. However, there are occasional regions devoid of strong vorticity near the jet edges, such as the engulfing previously noted, due to the intermittent nature of the turbulence near the jet edges.

Figure 7 shows the downstream variation of the three root-mean-square fluctuating vorticity components as well as the mean spanwise vorticity in the lower high-shear region, $y / \delta_{U} \approx-1.0$. In the region $0<x / h<3.0$ the mean spanwise vorticity decays while the vorticity fluctuations grow strongly. Downstream of $x / h=2.5$ all three components of the root-mean-square vorticity are greater than the mean vorticity. This coincides with the rapid increase in three-dimensionality of the vorticity contours observed in figure 4.

The root-mean-square of the centreline fluctuating vorticity (not shown) does not begin to grow until $x / h \approx 4.0$ after which there is strong growth in the region $4.0<x / h<7.0$. The spanwise vorticity fluctuation intensity begins to grow slightly earlier than the streamwise and lateral components, although, the centreline values for the three components are generally of the same magnitude. While all three components of the root-mean-square vorticity are of about the same magnitude on the jet centreline, in the high-shear region the streamwise vorticity fluctuations are consistently larger than the spanwise and lateral components.

In the region of strong vorticity growth and interaction between the two shear layers, $2.0 \leqslant x / h \leqslant 6.0$, there is a strong reorganization of the large-scale coherent structures present in the jet. Figure 8 shows the coherency spectra of the lateral 

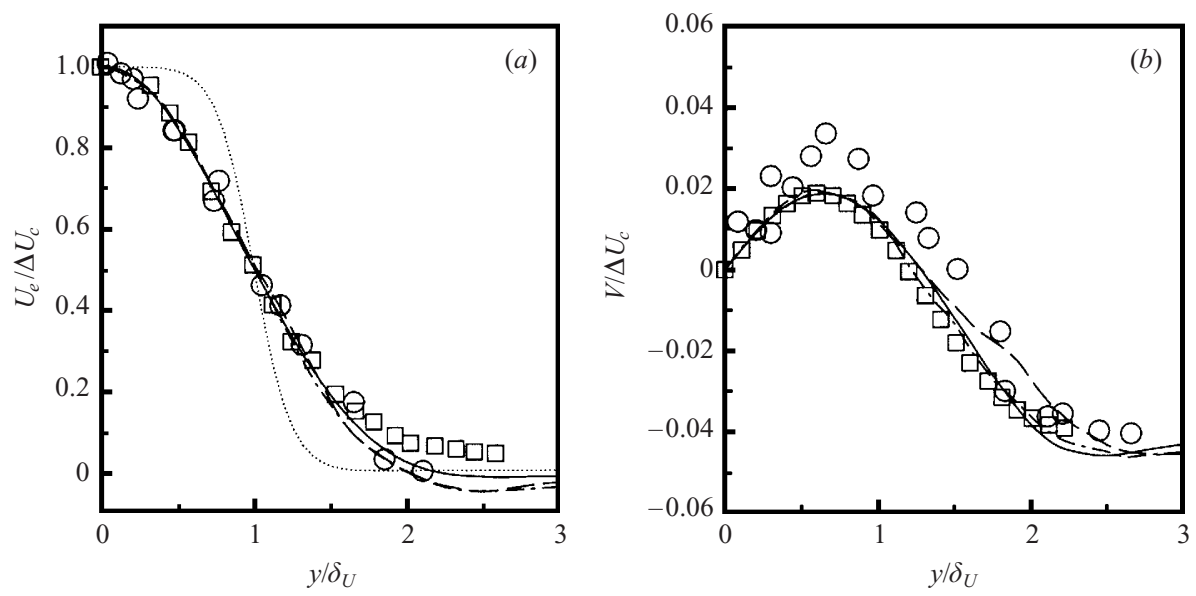

FIGURE 9. Mean velocity profiles in a planar jet. $\square$, Gutmark \& Wygnanski (1976); O, Ramaprian \& Chandrasekhara (1985). (a) Longitudinal velocity, $\cdots \cdots, x / h=0 ;-, x / h=4.0 ;---, x / h=9.0$; ,$-- x / h=11.0$. (b) Lateral velocity,,$- x / h=5.0 ;---, x / h=9.0 ;--, x / h=11.0$.

velocity at points on opposite sides of the jet at the stations $x / h=1.0,3.0$ and 5.0 with the frequency normalized using the local jet scaling. The coherency spectra are scaled using the inflow parameters to show the absolute growth of individual peaks. While the shear layer mode grows strongly between $x / h=0$ and $x / h=1.0$, as was shown in figure 3, downstream it is clear that the jet mode, $S=f \delta_{U} / \Delta U_{c}=0.11$, becomes dominant. At $x / h=5.0$ a strong peak has formed at frequencies near $S=f \delta_{U} / \Delta U_{c}=0.10$.

\subsection{Statistical description}

The statistics for the DNS were calculated by averaging in time as well as across the homogeneous $z$-direction in the jet. In addition, the symmetry of the jet was utilized to double the averaging sample.

Figure $9(a)$ shows profiles of the mean streamwise velocity excess, $U_{e}=U-U_{2}$, at several downstream stations. The mean profile at $x / h=0$ clearly shows the sharp shear layer at the edge of the jet. The streamwise velocity profiles from the three downstream stations shown, $x / h \geqslant 4.0$, collapse to a self-similar profile which compares well with the experimental data.

Figure $9(b)$ shows the mean profiles of the lateral velocity component at three stations in the jet. In a jet, the free-stream value of the mean cross-stream velocity, $V$, is equivalent to the entrainment velocity, $V_{e}$. The most upstream station shown, $x / h=5.0$, is the first station at which the entrainment velocity, $V_{e}$, has approached the magnitude of the self-similar value. Although the mean lateral profiles do not collapse as well using similarity scaling as the mean longitudinal profiles, they compare well with experimental data.

Figure 10 shows the downstream growth in the jet half-width based on the longitudinal velocity and the decay in the centreline velocity excess. The width of the jet is expressed as the half-width, $\delta_{U}$, defined as the distance from the jet centreline to the point at which the mean streamwise velocity excess is half of the centreline velocity excess. It can be seen that the jet growth and centreline velocity decay compare well with the experimental results. Analysis of the self-similar region of planar jets predicts 


\begin{tabular}{lcrcr}
\hline Source & $K_{1 u}$ & \multicolumn{1}{c}{$K_{2 u}$} & \multicolumn{1}{c}{$C_{1 u}$} & \multicolumn{1}{c}{$C_{2 u}$} \\
DNS results & 0.092 & 2.63 & 0.201 & 1.23 \\
Ramaprian \& Chandrasekhara (1985) & 0.110 & -1.00 & 0.093 & -1.60 \\
Browne et al. (1983) & 0.104 & -5.00 & 0.143 & -9.00 \\
Thomas \& Prakash (1991) & 0.110 & 0.14 & 0.220 & -1.20 \\
Thomas \& Chu (1989) & 0.110 & 0.14 & 0.220 & -1.19 \\
Gutmark \& Wygnanski (1976) & 0.100 & -2.00 & 0.189 & -4.72 \\
Hussain \& Clark (1977) & 0.118 & 2.15 & 0.123 & 4.47
\end{tabular}

TABLE 2. Jet growth rates and centreline velocity decay rates for the current results and several experimental studies.
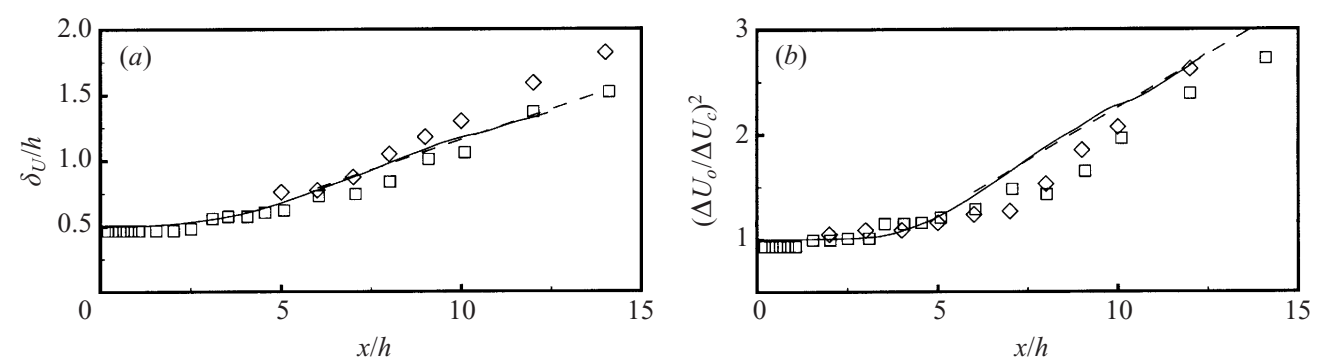

FIGURE 10. Downstream growth of the jet half-width and centreline mean velocity excess decay. - Current results; $\square$, Thomas \& Chu (1989); $\diamond$, Browne et al. (1983). (a) Jet half-width. - - -, $\delta_{U} / h=0.092(x / h+2.63) ;(b)$ Centreline mean velocity excess.,$---\left(\Delta U_{o} / \Delta U_{c}\right)^{2}=0.201(x / h+1.23)$.

a linear relationship between the jet width and the streamwise coordinate, $x$,

$$
\frac{\delta_{U}}{h}=K_{1 u}\left[\frac{x}{h}+K_{2 u}\right] \text {. }
$$

The current results give the values $K_{1 u}=0.092$ and $K_{2 u}=2.63$ for the region from $x / h=6.0$ to 12.0. As shown in table 2 , the thickness growth rate for the current results compares generally well with the experimental results, although they are somewhat lower. While the Reynolds numbers for these experimental studies vary, the selfsimilar growth rates are relatively consistent. There is a large variation in the virtual origins $K_{2 u}$ of the experimental studies which makes a comparison difficult. Since the virtual origins of the plane jet are strongly affected by the conditions at the nozzle (see Stanley \& Sarkar 2000) it is expected that these values will vary.

The analysis of planar jets predicts an inverse-squared relationship between the mean centreline velocity excess and the downstream coordinate, $x$,

$$
\left(\frac{\Delta U_{o}}{\Delta U_{c}}\right)^{2}=C_{1 u}\left[\frac{x}{h}+C_{2 u}\right]
$$

where $\Delta U_{o}$ is the centreline mean velocity excess at the jet nozzle and $\Delta U_{c}$ is the centreline mean velocity excess at the specific $x / h$ station. The current results predict values for the coefficients of $C_{1 u}=0.201$ and $C_{2 u}=1.23$. Table 2 shows a comparison of these values with results from several experimental studies. It is clear that there is a broad variation in the values of the centreline velocity decay rate as well as the virtual origins among the different studies. However, the current results are well within the range of values from the experimental data. 

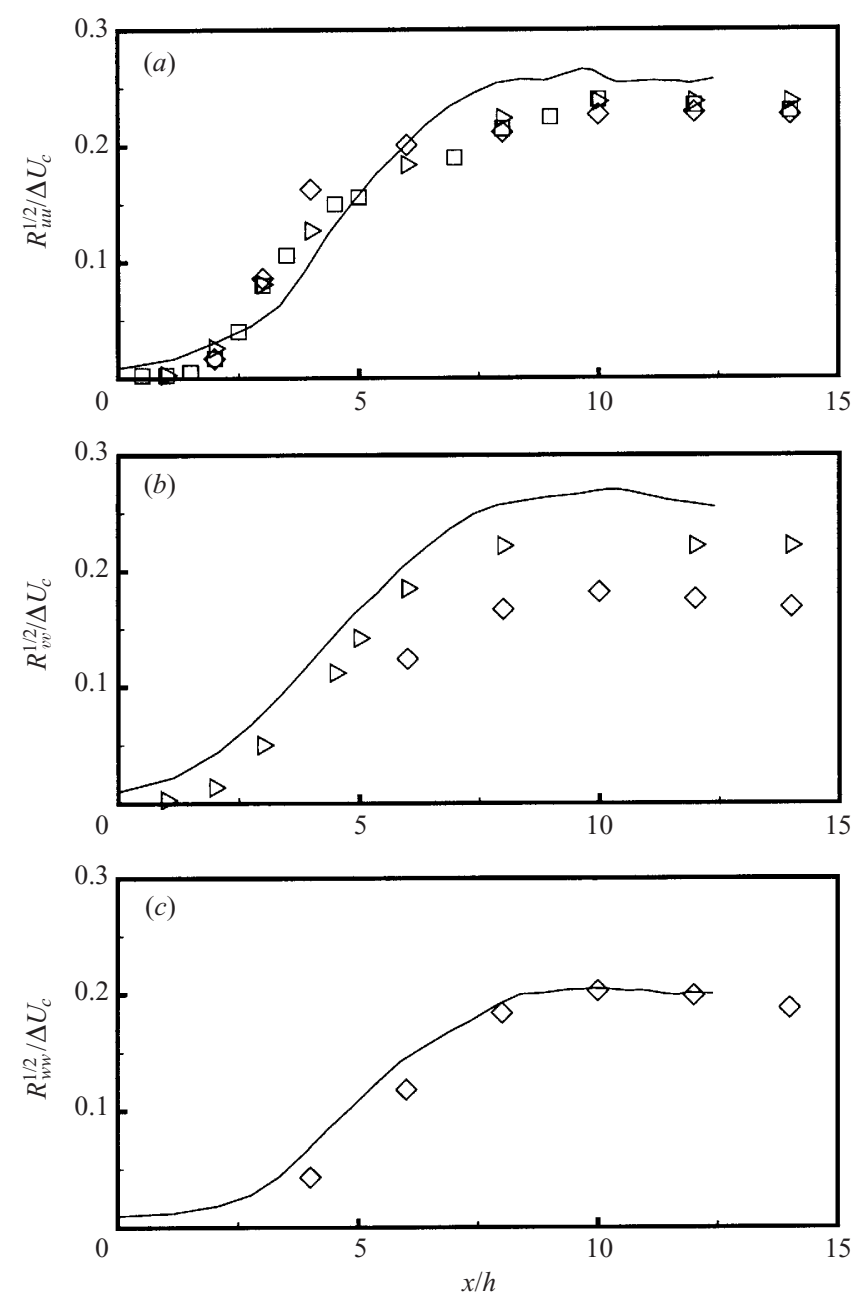

FIGURE 11. Growth of the normal Reynolds stresses on the jet centreline. - Current results; $\square$, Thomas \& Chu (1989); $\triangleright$, Thomas \& Prakash (1991); $\diamond$, Browne et al. (1983). (a) Longitudinal Reynolds stress. $(b)$ Lateral Reynolds stress. (c) Spanwise Reynolds stress.

The turbulent planar jet is a flow in which the mean Reynolds number, $R e_{\delta_{U}}=$ $2 \bar{\rho} \delta_{U} \Delta U_{c} / \mu$, grows downstream in the self-similar region as $\operatorname{Re}_{\delta_{U}} \propto x^{1 / 2}$. In the current simulation the mean Reynolds number remains nearly constant at the inflow Reynolds number of $R e_{h}=3000$ up to $x / h=1.5$. However, downstream of $x / h=2.0$, the mean Reynolds number grows strongly with the expected variation of $x^{1 / 2}$. At $x / h=12.0$, the mean Reynolds number has grown to a value of $R e_{\delta_{U}}=4838$.

Figure 11 shows the downstream evolution of the Reynolds stresses $R_{u u}, R_{v v}$, and $R_{w w}$ on the jet centreline. The fluctuation intensities on the jet centreline grow strongly in the region $2.0<x / h<10.0$. While the longitudinal and spanwise centreline intensities appear to asymptote to their self-similar values by $x / h=12.0$, the lateral intensity seems to be decaying towards its equilibrium in the final region.

It should be noted that the initial fluctuation intensities in the shear layers of the DNS case discussed here are somewhat high. The typical approach in experimental studies is to report the fluctuation intensity at the centreline of the nozzle, rather than 


\begin{tabular}{lcccc}
\hline Source & $\overline{u^{\prime} u^{\prime}} / \overline{q^{2}}$ & $\overline{v^{\prime} v^{\prime}} / \overline{q^{2}}$ & $\overline{w^{\prime} w^{\prime}} / \overline{q^{2}}$ & $\left|\overline{u^{\prime} v^{\prime}}\right| \overline{q^{2}}$ \\
DNS Results & 0.42 & 0.27 & 0.31 & 0.20 \\
Wygnanski \& Fiedler (1970) & 0.43 & 0.26 & 0.31 & 0.13 \\
Spencer \& Jones (1971) & 0.53 & 0.23 & 0.23 & 0.20 \\
Bell \& Mehta (1990) & 0.44 & 0.27 & 0.29 & 0.14 \\
TABLE 3. Comparison of the shear-layer turbulence intensities at $x / h=4.0$ with experimentally \\
measured values for fully developed turbulent shear layers. \\
\hline
\end{tabular}

in the shear layers. Since the fluctuation intensities peak in the boundary layers on the nozzle lips, the centreline intensity is not sufficient to quantify the inflow conditions of the jet. In the studies of Thomas \& Chu, Thomas \& Prakash and Browne et al. the centreline fluctuation intensity is on the order of $0.35 \%$, table 1 . However, the peak intensity in the shear layers from these studies is unknown. Likewise, the spectral content of the inflow fluctuations from these experiments is unknown. The centreline fluctuation intensity for the current study is $1.9 \%$ while the peak intensity in the shear layers is approximately $10 \%$ of the jet nozzle velocity.

The initial rate of growth of the fluctuations at the jet centreline is consistent with the experimental studies. The initial growth rate of the longitudinal fluctuation intensity in the region $2.0<x / h<5.0$ is a little low, figure 11(a). As discussed in Stanley \& Sarkar (2000), the initial growth of turbulence intensities is weaker for thicker shear layers and therefore the slower growth in the simulation may be related to the smaller $h / \theta=20$ in the DNS relative to experimental values, see table 1 . In the experiments, the longitudinal fluctuations grow more rapidly near the inflow than the lateral or spanwise components. In the current results, longitudinal and spanwise components of the fluctuating velocity field grow at approximately the same rate on the jet centreline, while the growth of the lateral Reynolds stress is stronger. This behaviour is probably due to the difference in the inflow fluctuation intensities and spectra between the current study and the experimental results. The forcing at the inlet can accentuate anisotropies that remain for a certain distance downstream. In shear flows, the longitudinal fluctuation intensities grow due to the production by the mean shear before the transfer of energy to the lateral and spanwise components can occur. The redistribution of energy is larger in nonlinearly evolving turbulent flows than in transitional or rapidly distorted flows. Therefore the transfer of energy from the longitudinal component to the lateral and spanwise components occurs more rapidly in the DNS where there is initially energy in all three velocity components at a level which is larger than that in experiments.

The direct production of energy by the mean shear into the $R_{u u}$ component in the sharp shear layers, $y / \delta_{U}= \pm 1.0$, for $x / h \leqslant 2.0$ leads to a strong anisotropy in the fluctuating fields in this region of the jet. At the station $x / h=2.0$ (not shown), the longitudinal component is about three times the lateral component and more than two times the spanwise component, leading to anisotropy in the normal components which is far greater than is present in the fully developed region of turbulent shear layers. However the shear component of the anisotropy $\overline{u^{\prime} v^{\prime}} / \overline{q^{2}}= \pm 0.20$ agrees quite well with experimentally measured values in turbulent shear layers.

In the region $2.0 \leqslant x / h \leqslant 4.0$ the anisotropy in the shear layers relaxes to values which are more characteristic of those present in turbulent shear layers. Table 3 shows the anisotropy values in the shear layers at the station $x / h=4.0$ along with 

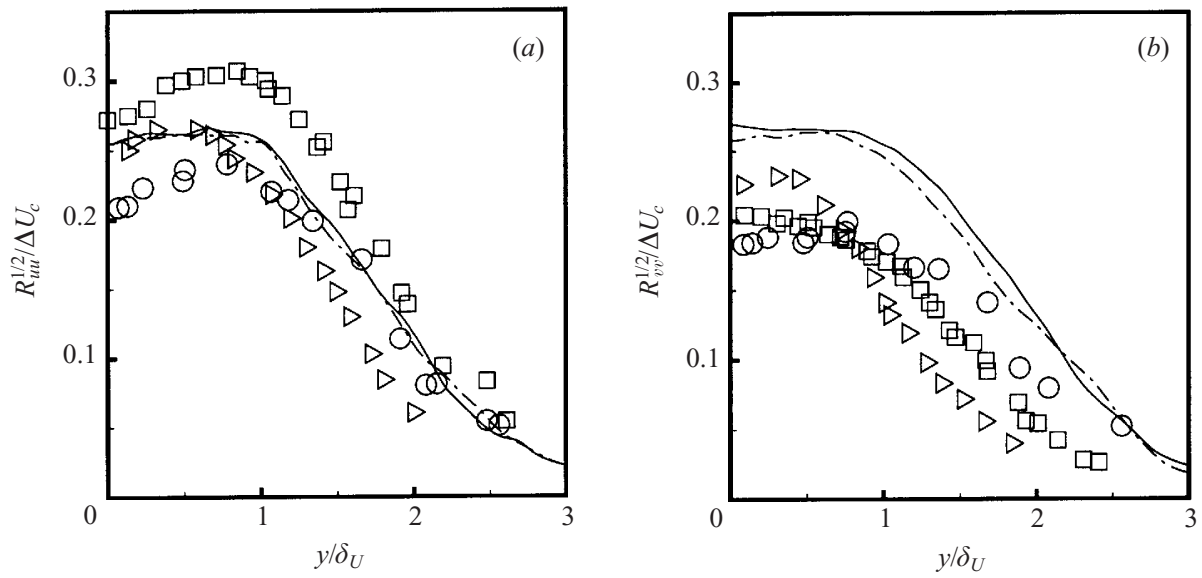

FiguRE 12. (a) Longitudinal and $(b)$ lateral Reynolds stress profiles downstream in the jet. $x / h=10.5 ;---x / h=12.0 ; \square$, Gutmark \& Wygnanski (1976); O, Ramaprian \& Chandrasekhara (1985); $\triangleright$, Thomas \& Prakash (1991).
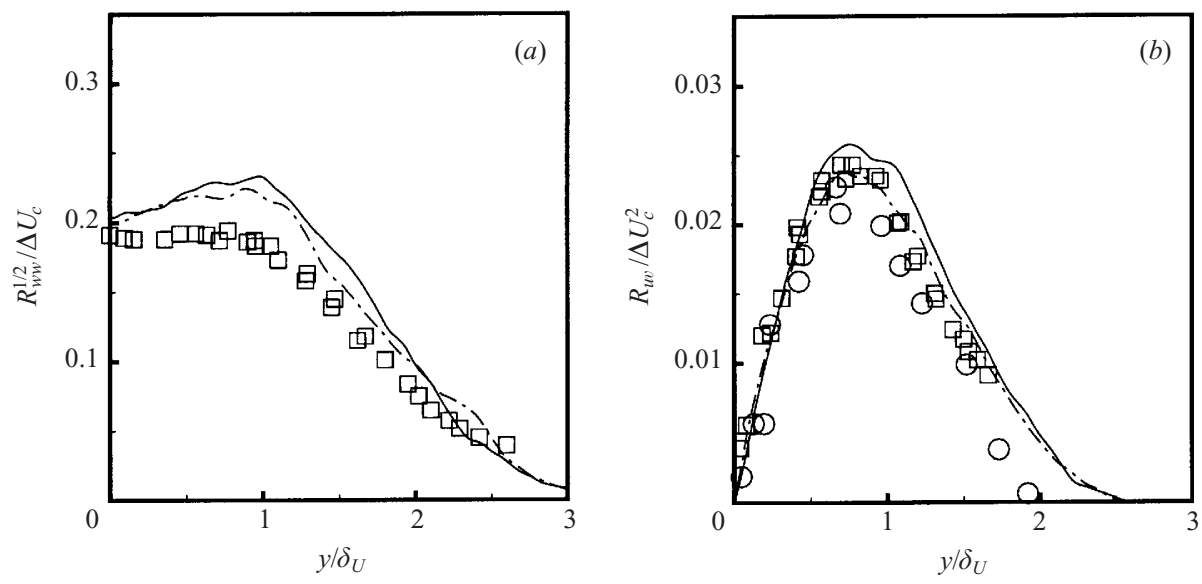

FIGURE 13. (a) Spanwise Reynolds stress and $(b)$ Reynolds shear stress profiles downstream in the jet. $\longrightarrow, x / h=10.5 ;---, x / h=12.0 ; \square$, Gutmark \& Wygnanski (1976); O, Ramaprian \& Chandrasekhara (1985).

experimental values for fully developed turbulent shear layers. At this station there is still an imbalance between the lateral and spanwise components; however, they have both become more comparable with the longitudinal component of the Reynolds stress. The ordering of the intensities is as would be expected, for a turbulent shear flow with the mean shear in the $y$-direction, with $\overline{u^{\prime} u^{\prime}} / \overline{q^{2}}>\overline{w^{\prime} w^{\prime}} / \overline{q^{2}}>\overline{v^{\prime} v^{\prime}} / \overline{q^{2}}$.

Figures 12 and 13 show the profiles of the Reynolds stress components downstream in the jet near the outflow of the domain. From the collapse of the profiles from the current results it can be seen that the Reynolds stress components are close to selfsimilarity near the outflow of the domain. Except for the lateral component, the other two fluctuation intensities as well as the Reynolds shear stress compare reasonably well with the experimental results. As can be seen, there is considerable scatter in the available experimental data. The downstream stations at which these experimental data were collected varies: $x / h=14$ for Thomas $\&$ Prakash, $x / h=40$ for Ramaprian 


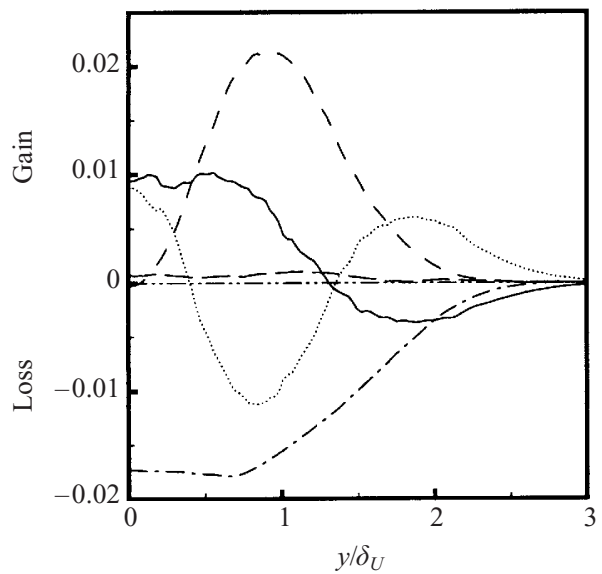

FiguRE 14. Balance of the terms in the turbulent kinetic energy equation in the fully developed region of the jet. $\longrightarrow$, Advection; - - , $P^{*} ;---,-\epsilon^{*} ; \cdots \cdots,-\partial_{j} T_{j}^{*} / \bar{\rho} ;----, \Pi^{*} / \bar{\rho} ;--, \partial_{t} K^{*}$.

\& Chandrasekhara and $x / h=140$ for Gutmark \& Wygnanski. For all three experimental studies the profiles at adjacent stations collapsed indicating a measure of self-similarity (even for Thomas \& Prakash at the downstream station of $x / h=14.0$ ). The lateral Reynolds stress is about $20 \%$ higher, and this is a consequence of the particular inflow forcing conditions, which happen to induce high two-dimensionality in the development region. The apparent sensitivity of the downstream fluctuation intensities to inflow conditions has been observed previously. For temporally evolving shear layers, Rogers \& Moser (1994) observed a strong increase in the lateral fluctuation intensity at long times due to the addition of a strong two-dimensional forcing. With weak forcing, the influence on the self-similar fluctuation intensities was more moderate. In temporally evolving plane wakes, however, Moser et al. (1998) observed a strong influence on the self-similar longitudinal and lateral fluctuation intensities due to weak forcing.

Figure 14 shows the balance of the terms in the turbulent-kinetic-energy equation in the fully developed region of the jet. Profiles in the range $9.5 \leqslant x / h \leqslant 11.0$ are obtained using similarity variables and averaged to obtain this balance. The trace of the pressure-strain term, $\Pi^{*}=\overline{p^{\prime} \partial_{k} u_{k}^{\prime}}$, also called the pressure-dilatation, is insignificant. Here, a star as super-index denotes normalization by $\Delta U$ and $\delta_{U}$. The strong production, $P^{*}=-R_{j k} \partial_{k} \tilde{u}_{j}$, of turbulent energy in the regions of peak shear, $y / \delta_{U} \approx \pm 0.8$, as well as the more uniform strong viscous dissipation of energy, $\epsilon^{*}=\overline{\tau_{j k}^{\prime} \partial_{k} u_{j}^{\prime}} /(\operatorname{Re} \bar{\rho})$, can be seen in the core of the jet, $y / \delta_{U} \leqslant 1$. The advection term in the turbulent-kinetic-energy equation acts to transport energy from the edges of the jet in towards the centreline while the turbulent transport term, $\partial_{j} T_{j}^{*}=\partial_{j}\left(\overline{\rho u_{k}^{\prime \prime} u_{k}^{\prime \prime} u_{j}^{\prime \prime}} / 2+\overline{u_{k}^{\prime} p^{\prime}} \delta_{k j}-\overline{u_{k}^{\prime} \tau_{j k}^{\prime}} / R e\right)$, acts to transport fluctuating energy away from the region of peak production towards the jet centreline as well as the jet edges. It is clear from the fact that the production term is zero at the jet centreline that the action of the advection and turbulent transport terms are the sole means by which fluctuating energy is present at the core of the jet.

Near the outer edges of the jet, as discussed by Tennekes \& Lumley (1972), the balance is predominately between the turbulent transport term and the advection. At this point in the jet, the turbulent transport term acts to spread turbulent energy outwards towards the jet edges while the advection due to the entrainment velocity, 


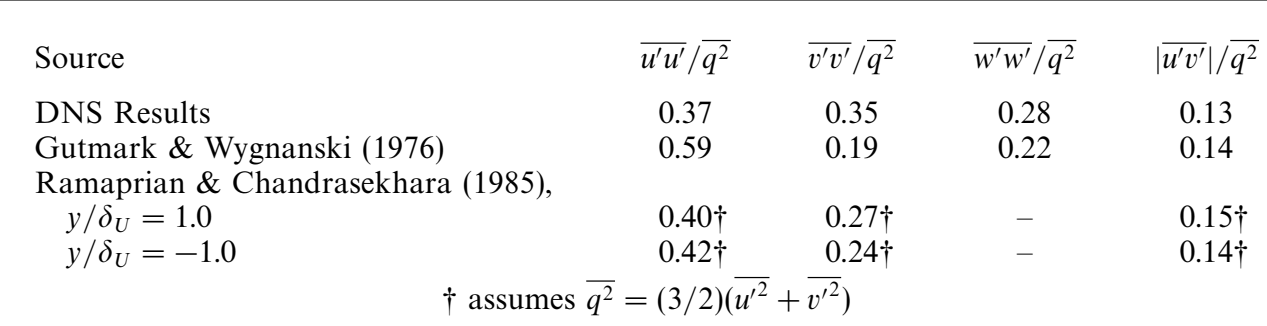

TABLE 4. Comparison of the jet turbulence intensities for $y / \delta_{U}= \pm 1.0$ in the fully developed region with experimentally measured values.

\begin{tabular}{lccc} 
Source & $\overline{u^{\prime} u^{\prime}} / \overline{q^{2}}$ & $\overline{v^{\prime} v^{\prime}} / \overline{q^{2}}$ & $\overline{w^{\prime} w^{\prime}} / \overline{q^{2}}$ \\
DNS Results & 0.37 & 0.39 & 0.23 \\
Gutmark \& Wygnanski (1976) & 0.48 & 0.27 & 0.24 \\
Browne et al. (1983) & 0.47 & 0.24 & 0.29 \\
Ramaprian \& Chandrasekhara (1985) & $0.37 \dagger$ & $0.29 \dagger$ & - \\
Everitt \& Robins (1978) & 0.38 & 0.34 & 0.28 \\
\multicolumn{1}{c}{$\dagger$ assumes $\overline{q^{2}}=(3 / 2)\left(\overline{u^{\prime 2}}+\overline{v^{\prime 2}}\right)$} &
\end{tabular}

TABLE 5. Comparison of the jet turbulence intensities on the centreline in the fully developed region with experimentally measured values.

$V_{e}$, transports energy inwards. It is the balance between these two terms which results in the edge of the turbulent jet being stationary in the mean.

In general, the turbulent-energy balance for the current results compares well with the energy balances shown by both Ramaprian \& Chandrasekhara (1985) and Gutmark \& Wygnanski (1976). However, a strong advantage of the current results in the study of the energy balance is that every term in the balance can be calculated. In experimental studies in the past, it has been necessary to make various approximations regarding the terms in the turbulent-kinetic-energy equation in order to estimate their balance. Ramaprian \& Chandrasekhara did not measure the spanwise velocity component, therefore they were forced to use an estimate of the turbulent kinetic energy based on the lateral and streamwise velocities only. Also, rather than measuring the dissipation directly, they estimated dissipation using the energy spectra of $u^{\prime 2}$. Gutmark \& Wygnanski, on the other hand, estimated the dissipation using the isotropic assumption, $\epsilon=15 v \overline{\left(\partial u^{\prime} / \partial x\right)^{2}}$. In general, all of the studies have restricted the form of the turbulent-kinetic-energy equation using the boundary-layer approximation for the mean velocity profiles to drop terms. While it is recognized that these are likely good and necessary approximations, in some cases the higher-order effects may be significant.

The Reynolds stress anisotropy levels for $y / \delta_{U}= \pm 1.0$ in the self-similar region of the jet are shown in table 4 compared to available experimental data. The results from this DNS show greater isotropy of the normal stress components than the experimental data of Ramaprian \& Chandrasekhara and Gutmark \& Wygnanski. The magnitudes of $\overline{u^{\prime} u^{\prime}} / \overline{q^{2}}$ and $\overline{v^{\prime} v^{\prime}} / \overline{q^{2}}$ are much more balanced in the self-similar region of the jet than was the case in the shear layers near the inflow. In both the DNS and experimental data the shear component of the isotropy, $\overline{u^{\prime} v^{\prime}} / \overline{q^{2}}$, is at a level consistent with those observed in turbulent shear layers, table 3 .

At the jet centreline, table 5, the large-scale isotropy does not change drastically 


\begin{tabular}{|c|c|c|c|}
\hline & $\underline{\left(\partial u^{\prime} / \partial x\right)^{2}}$ & $\left(\partial v^{\prime} / \partial y\right)^{2}$ & $\left(\partial w^{\prime} / \partial z\right)^{2}$ \\
\hline & $\overline{(\epsilon / 15 v)}$ & $(\epsilon / 15 v)$ & $\overline{(\epsilon / 15 v)}$ \\
\hline lue & 1.0 & 1.0 & 1.0 \\
\hline$y / \delta_{U}= \pm 1.0$ & 0.883 & 1.034 & 1.095 \\
\hline Centreline & 1.014 & 0.975 & 1.019 \\
\hline
\end{tabular}

TABLE 6. Normal-derivative variances in the self-similar region of the jet.

\begin{tabular}{|c|c|c|c|c|c|c|}
\hline & $\overline{\frac{\left(\partial u^{\prime} / \partial y\right)^{2}}{\left(\partial u^{\prime} / \partial x\right)^{2}}}$ & $\overline{\frac{\left(\partial u^{\prime} / \partial z\right)^{2}}{\left(\partial u^{\prime} / \partial x\right)^{2}}}$ & $\overline{\frac{\left(\partial v^{\prime} / \partial x\right)^{2}}{\left(\partial v^{\prime} / \partial y\right)^{2}}}$ & $\overline{\left(\partial v^{\prime} / \partial z\right)^{2}} \frac{\left(\partial v^{\prime} / \partial y\right)^{2}}{}$ & $\overline{\frac{\left(\partial w^{\prime} / \partial x\right)^{2}}{\left(\partial w^{\prime} / \partial z\right)^{2}}}$ & $\overline{\frac{\left(\partial w^{\prime} / \partial y\right)^{2}}{\left(\partial w^{\prime} / \partial z\right)^{2}}}$ \\
\hline lues & 2.0 & 2.0 & 2.0 & 2.0 & 2.0 & 2.0 \\
\hline$y / \delta_{U}= \pm 1.0$ & 2.36 & 2.30 & 1.58 & 2.07 & 1.61 & 2.20 \\
\hline Centreline & 2.06 & 2.13 & 1.95 & 1.96 & 1.95 & 1.91 \\
\hline
\end{tabular}

TABLE 7. Cross-derivative variances in the self-similar region of the jet.

from the levels present in the high-shear region. The lateral component is higher than the isotropic level of $1 / 3$ while the spanwise component is below this level. It is clear that the large scales have not relaxed to isotropy in spite of the absence of mean shear at the centreline. This is consistent with the experimental data shown in the table although, as in the high-shear region, there is a large variation in the level of anisotropy, with Gutmark \& Wygnanski having the highest levels and Ramaprian \& Chandrasekhara and Everitt \& Robins having more isotropic values consistent with the DNS results.

While it is clear that the large scales of the flow relax slowly to isotropy in the absence of mean shear at the centreline, the small scales adjust quite rapidly. Table 6 lists the normal velocity derivative variances for the centreline as well as the highshear regions in the self-similar region of the jet near the outflow. In the high-shear regions, above and below the centreline, the deviation with respect to the isotropic condition that $\overline{\left(\partial u^{\prime} / \partial x\right)^{2}}=\overline{\left(\partial v^{\prime} / \partial y\right)^{2}}=\overline{\left(\partial w^{\prime} / \partial z\right)^{2}}$ is as large as $12 \%$ while at the centreline of the jet this condition is met to within $3 \%$. This is an indication that the isotropic estimate for the dissipation would perform well at the centreline while a small error of order $10 \%$ would be present at the shear zone.

As shown in table 7, to a close approximation, the cross-derivative variances of the longitudinal velocity, $\overline{\left(\partial u^{\prime} / \partial y\right)^{2}}$ and $\overline{\left(\partial u^{\prime} / \partial z\right)^{2}}$, are nearly equal at both the centreline and in the shear region. Although different from the isotropic estimate in the shear regions, the ratio of these terms to the normal derivative variance, $\overline{\left(\partial u^{\prime} / \partial x\right)^{2}}$, approaches the isotropic value of 2.0 near the centreline. The cross-derivative variances of the lateral and spanwise velocity components are more affected by the mean shear than those of the longitudinal velocity, so that $\overline{\left(\partial v^{\prime} / \partial x\right)^{2}} \neq \overline{\left(\partial v^{\prime} / \partial z\right)^{2}}$ and $\overline{\left(\partial w^{\prime} / \partial x\right)^{2}} \neq \overline{\left(\partial w^{\prime} / \partial y\right)^{2}}$ in the regions of strong shear. However, near the centreline these conditions are met quite well and the ratios of these cross-derivative variances to their respective normal-derivative variances match the isotropic value of 2.0 within $9 \%$. It is clear from these data that the small scales of motion in the self-similar region of the jet adjust rapidly, in space, to the local mean velocity profile so that near the centreline of the jet the small scales are, to a relatively good approximation, isotropic.

Integral length scales were calculated in the fully developed region of the jet from the 

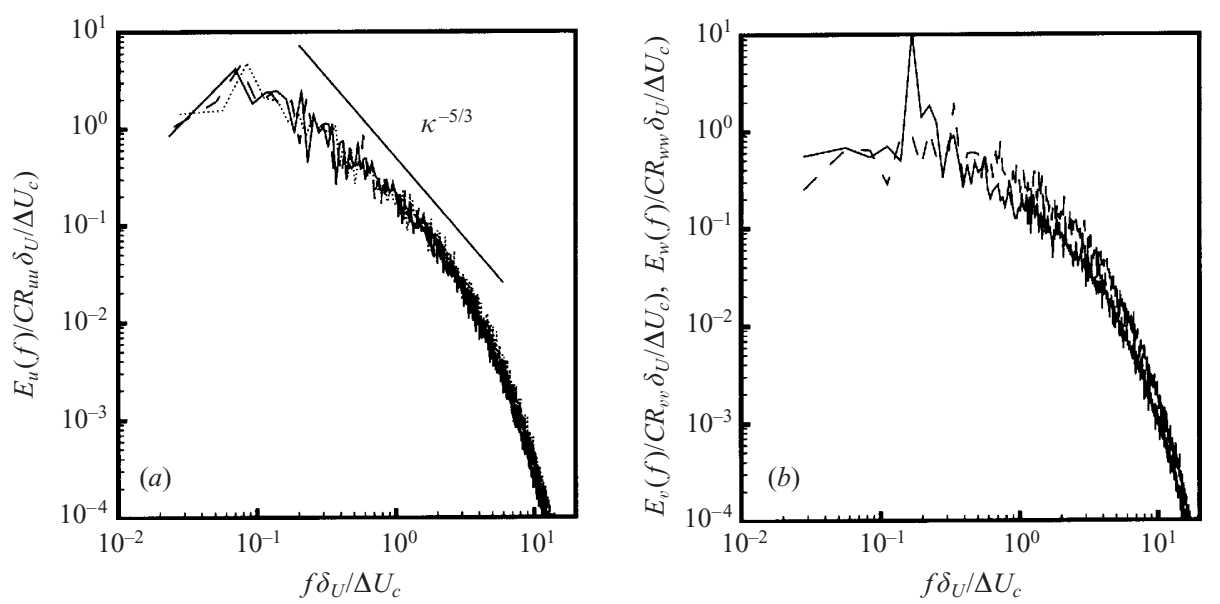

FIGURE 15. One-dimensional autospectra on the centreline of the jet. (a) Longitudinal velocity autospectra. $\longrightarrow, x / h=10.0 ;---, x / h=11.0 ; \cdots \cdots, x / h=12.0$. $(b)$ Lateral and spanwise velocity autospectra at $x / h=12.0$.,$- E_{v}(f) ;---, E_{w}(f)$.

corresponding correlations. The values obtained were $\Lambda_{x} / \delta_{U}=0.65, \Lambda_{y} / \delta_{U}=0.37$ and $\Lambda_{z} / \delta_{U}=0.22$. The longitudinal integral length scale from the DNS results is a little high compared with the experimentally measured values of $\Lambda_{x} / \delta_{U}=0.47$ by Gutmark \& Wygnanski and $\Lambda_{x} / \delta_{U}=0.35$ measured by Everitt \& Robins. Nevertheless, the ratio of the longitudinal to lateral scales, $\Lambda_{x} / \Lambda_{y}=1.75$, is within the range observed in the experiments. Gutmark \& Wygnanski reported a value of 2.14 while Everitt \& Robins reported 1.59 .

Figure 15(a) shows the one-dimensional autospectra in time of the longitudinal velocity on the centreline of the jet at three stations downstream in the self-similar region. These spectra are scaled using the local values of the jet width, centreline velocity excess and the centreline variance of the longitudinal velocity, so that the integral between zero and infinity of these non-dimensional quantities is unity. It can be seen in this figure that, for the conditions of this jet, all of the spectra collapse relatively well. The energy spectra began exhibiting signs of self-similar behaviour in the region near $x / h=9.0$. The $k^{-5 / 3}$ law is plotted for comparison, although for the conditions of this simulation an extended inertial subrange is not observed in the energy spectra. However, there is clearly a dissipative region at high frequencies where the energy spectra decay at greater than a $k^{-5 / 3}$ rate.

Consistent with the earlier observation about the relative isotropy of the small scales in the fully developed region of the jet, the autospectra in time of the lateral and spanwise velocity components are very similar for the small-scale, high-frequency motions, figure 15(b). However, as noted previously, the large-scale, low-frequency, motions of two velocity components are not similar. A strong peak occurs in the autospectra of the lateral velocity component near the jet mode, $S=f \delta_{U} / \Delta U_{c} \approx 0.11$. Due to the symmetries associated with the isotropic turbulence assumption, the autospectra of the lateral and spanwise velocity components would be identical if the velocity fields were completely isotropic.

Figure 16 shows an estimate of the dissipation spectra on the jet centreline at three downstream stations in the jet. For this figure, the dissipation spectra are estimated as $f^{2} E_{u}(f)$ where $E_{u}(f)$ is the one-dimensional autospectrum in time shown in figure 15(a). This figure clearly indicates that there is a strong peak in the 


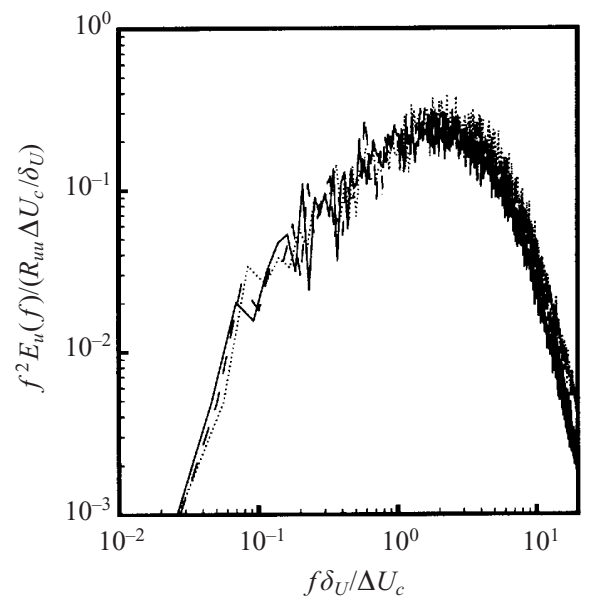

FIGURE 16. One-dimensional dissipation spectra in time of the longitudinal velocity on the centreline in the self-similar region.,$- x / h=10.0 ;---, x / h=11.0 ; \cdots \cdots, x / h=12.0$.

dissipation spectra with more than an order-of-magnitude decay at higher frequencies. This, coupled with the three to four orders-of-magnitude decay present in both the temporal and the spanwise autospectra, are a strong indication that the results in this study are well resolved.

\section{Scalar evolution and mixing}

The evolution of the passive scalar field in a $R e_{h}=3000$ turbulent jet is discussed in this section. The physical conditions for this simulation match those of the previous jet except for a decrease in the inflow forcing intensity to $5 \%$ in the shear layers. The physical conditions for the experimental studies utilized herein are given in table 1 .

In the course of the discussion of the evolution of the passive scalar, comparison will be made to the experimental data of Ramaprian \& Chandrasekhara (1985), Browne et al. (1983), Davies, Keffer \& Baines (1975) and Jenkins \& Goldschmidt (1973). In these studies the evolution of the 'scalar' field was studied through experimental measurements of heated jets in air and water and the temperature difference between the jet and free-stream fluids was kept small in order to minimize the effects of buoyancy on the evolution of the jet. Molecular effects on the evolution correspond to those of a $\mathrm{Pr}=O(1)$ scalar. The Schmidt number used for the passive scalar in this jet is $S c=1.0$.

\subsection{Structural development}

Figures 17 and 18 show instantaneous isocontours of the passive scalar on an $(x, y)$-plane (side section) and a $(x, z)$-plane (top section) in the jet, respectively, at a non-dimensional time $t \Delta U_{o} / h=89.10$. The $(x, z)$-plane shown is at a station $y / h=0.467$ which is just below the upper shear layer at the inflow. At this lateral station the fluid at the inflow is predominately jet fluid. In these figures, the contour levels are defined such that white indicates pure coflow fluid, $\tilde{\xi}=0$, while black indicates pure jet fluid, $\tilde{\xi}=1.0$. The levels of grey in between are a measure of the mixedness of the fluid with darker indicating proportionally large quantities of jet fluid. The dark core of pure jet fluid is clearly evident in figure 17 near the inflow 


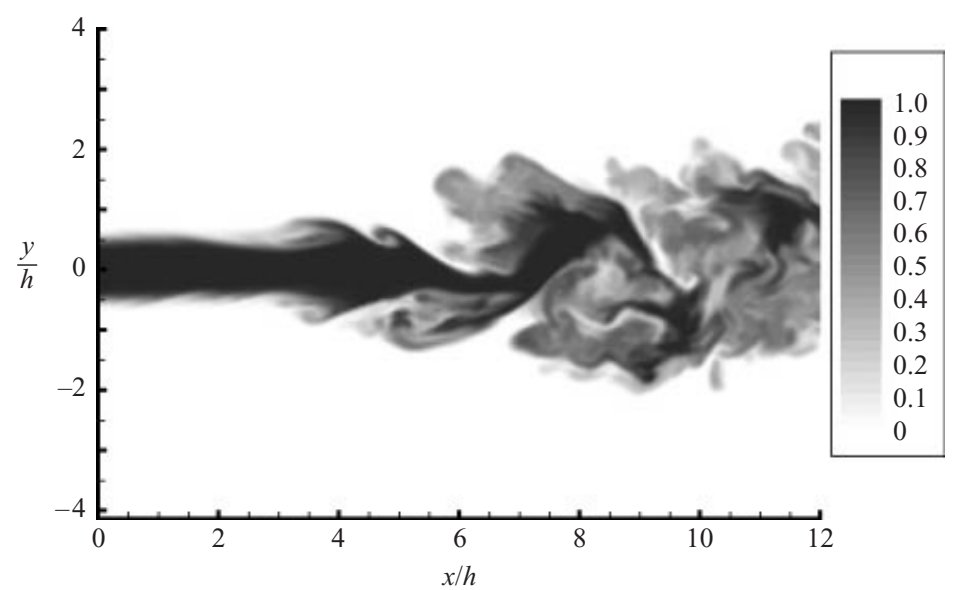

FIGURE 17. Instantaneous passive scalar contours on an $(x, y)$-plane, $z=0$.

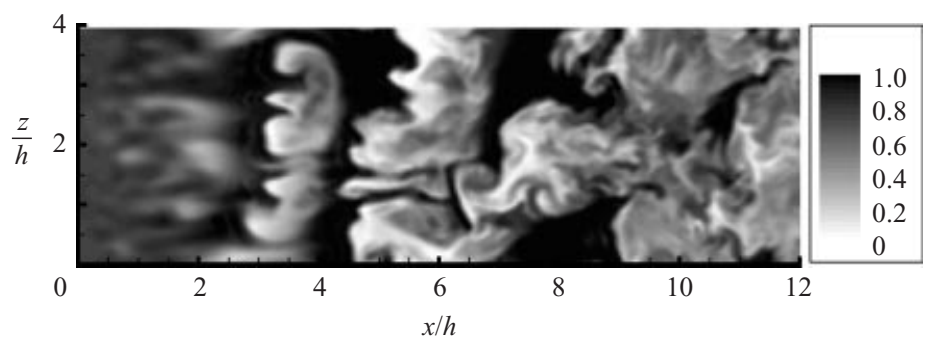

FIGURE 18. Instantaneous passive scalar contours on a $(x, z)$-plane just below the upper shear layer, $y / h=0.44$.

plane. In the region $x / h<4.0$ there is a slight spreading of the core of the jet due to the effects of turbulent diffusion in the shear layers. At $x / h=4.0$ a large-scale rollup is present in the upper and lower shear layers. The large-scale entrainment of coflow fluid by the upper structure is evident in figure 18 by the sudden appearance of a large region of white at $3.0 \leqslant x / h \leqslant 4.0$. While not entirely two-dimensional, as indicated by the inclination and spanwise inhomogeneity, the spanwise extent of this structure is large. In the region $5.0 \leqslant x / h \leqslant 7.0$ the remnants of a second structure can be seen in figures 17 and 18. As is evidenced by the medium grey shading of this structure in figure 18 the effects of small-scale mixing have eliminated any region of pure coflow fluid entrained by this structure. Likewise, this structure has greater three-dimensionality than that of the structure nearer to the inflow.

Downstream in the jet, $x / h \geqslant 8.0$, the effects of small-scale mixing due to the strong turbulence in the jet have greatly reduced the presence of patches of pure jet and coflow fluid in the jet. However, there are small regions which are predominantly composed of one fluid type or the other. In figure 17 there is clearly a small black patch composed primarily of jet fluid present near the jet centreline at $x / h=11.0$. Likewise, near the jet edges there are clearly regions where nearly pure coflow fluid have been engulfed into the more mixed fluid present in the jet.

\subsection{Statistical description}

Figure 19 shows the mean profiles of the passive scalar field at several streamwise stations normalized using the centreline mean scalar value, $\tilde{\xi}_{c}$. At the inflow plane, 


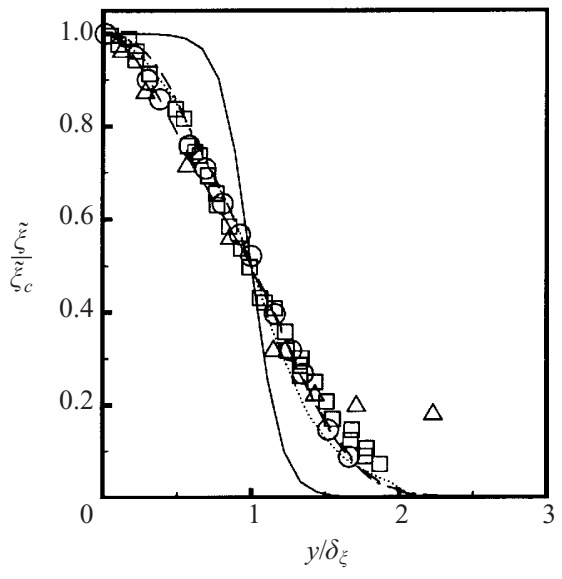

FiguRE 19. Mean scalar profiles in a planar jet.,$- x / h=0 ;---, x / h=7.0 ;---, x / h=10.0$; $\cdots \cdots, x / h=12.0 ; \square$, Davies et al. (1975); $\triangle$, Ramaprian \& Chandrasekhara (1985); O, Jenkins \& Goldschmidt (1973).

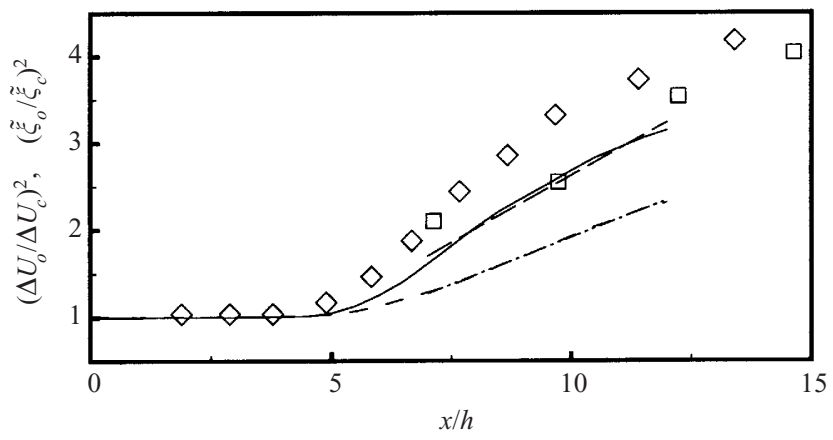

FIGURE 20. Decay of the mean scalar and centreline velocity excess on the jet centreline $-\left(\tilde{\xi}_{o} / \tilde{\xi}_{c}\right)^{2} ;---,\left(\Delta U_{o} / \Delta U_{c}\right)^{2} ;--,\left(\tilde{\xi}_{o} / \tilde{\xi}_{c}\right)^{2}=0.308(x / h-1.48) ;---,\left(\Delta U_{o} / \Delta U_{c}\right)^{2}=$ 0.213(x/h-1.02); $\diamond$, Browne et al. (1983); $\square$, Davies et al. (1975).

$x / h=0$, the sharp transition from pure coflow fluid, $\tilde{\xi}=0$, to pure jet fluid, $\tilde{\xi}=1.0$, in the near field of the jet is evident. Downstream, the effects of strong turbulent mixing in the shear layers act to spread this profile until self-similarity is approached near $x / h=7.0$. The mean passive scalar profiles are slightly slower to develop than the mean velocity field. The mean longitudinal velocity becomes approximately selfsimilar at $x / h=10.0$ in this jet. Due to the lower fluctuation intensity at the inflow, this jet develops slower than that discussed in $\S 5$.

The self-similar scaling of the mean profiles in figure 19 masks the mixing and subsequent decay in the overall scalar values downstream in the jet. The decay in the mean value of the passive scalar on the jet centreline provides a measure of the overall mixing in the jet. Figure 20 shows the downstream variation in the centreline scalar values plotted using self-similar scaling. The symbols in this figure are experimental data for the centreline temperature decay in heated jets. The current results show a strong initial decay followed by a slower decay downstream, similar to the results of Browne et al.

The mathematical analysis for the self-similar region of the planar jet gives a downstream variation of the mean scalar on the centreline of $\tilde{\xi}_{c} \propto x^{-1 / 2}$ similar to 


\begin{tabular}{lcccrcc}
\hline Source & $K_{1 \xi}$ & $K_{2 \xi}$ & $C_{1 \xi}$ & $C_{2 \xi}$ & $K_{1 u} / K_{1 \xi}$ & $C_{1 u} / C_{1 \xi}$ \\
DNS, $q / \Delta U_{o}=0.05$ & 0.158 & -1.34 & 0.308 & -1.48 & 0.66 & 0.69 \\
Ramaprian \& Chandrasekhara (1985) & 0.167 & 2.00 & 0.194 & 6.00 & 0.66 & 0.87 \\
Browne et al. (1983) & 0.128 & 5.00 & 0.189 & 7.86 & 0.81 & 0.76 \\
Jenkins \& Goldschmidt (1973) & 0.123 & 0.090 & 0.261 & -5.62 & 0.71 & 0.61 \\
Davies et al. (1975) & 0.115 & 2.05 & 0.258 & 0.920 & 0.87 & 0.61
\end{tabular}

TABLE 8. Jet growth rates based on the passive scalar and centreline scalar decay rates for the current results and several experimental studies.

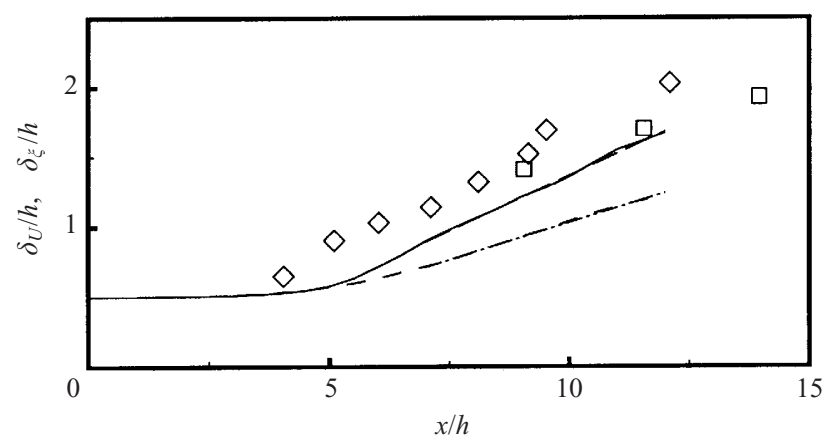

FiguRE 21. Downstream growth of the jet half-widths based on the passive scalar and velocity. $\delta_{\xi} / h ;---, \delta_{U} / h ;--, \delta_{\xi} / h=0.158(x / h-1.34) ;---, \delta_{U} / h=0.105(x / h-0.220) ; \diamond$, Browne et al. (1983); $\square$, Davies et al. (1975).

the decay of the centreline mean velocity excess (5.3). Table 8 shows the fit of the current data in the region $x / h \geqslant 7.0$ as well as the experimentally obtained fits. In general, the centreline scalar decay rates from the DNS are high compared to the experimental values. It is reasonable that the decrease in the slope of the DNS scalar decay is a gradual shift into a slower more linear decay downstream.

Figure 21 shows the downstream growth in the jet half-width based on the mean profiles of the passive scalar. The slow initial development of this jet to the linear growth rate, $\delta_{\xi} \propto x$, anticipated in the self-similar region is apparent. While Browne et al. (1983) see linear growth near $x / h=3.0$, for the current case linear growth is not observed until $x / h=6.0$. However, as can be seen in table 8 , the linear growth rate, $K_{1 \xi}$, based on the region $x / h \geqslant 7.0$ compares well with the experimental data for heated jets. As with the virtual origins for the velocity field, there is a great deal of scatter in the values for the virtual origins of the scalar field, $K_{2 \xi}$ and $C_{2 \xi}$ due to variations in the jet nozzle conditions between the various studies.

The decay of the centreline mean velocity excess and the jet half-width based on the velocity field for this case are also shown in figures 20 and 21 . The constants in the fits for the self-similar development of $\delta_{U} / h$ and $\left(\Delta U_{o} / \Delta U_{c}\right)^{2}$ are $K_{1 u}=0.105$, $K_{2 u}=-0.220, C_{1 u}=0.213$ and $C_{2 u}=-1.02$, based on the region $x / h \geqslant 7.0$. The spread rates and centreline decay rates for the scalar field are larger than those for the velocity field, indicating that turbulent scalar transport occurs at a faster rate than turbulent momentum transport. The ratios of scalar to velocity spread rates, $K_{1 u} / K_{1 \xi}=0.66$, and scalar to velocity centreline decay rates, $C_{1 u} / C_{1 \xi}=0.69$, are in the range of experimental values for the temperature field, table 8 , and are consistent with the turbulent Schmidt number, $S c_{t}=v_{t} / D_{t} \approx 0.7$. 


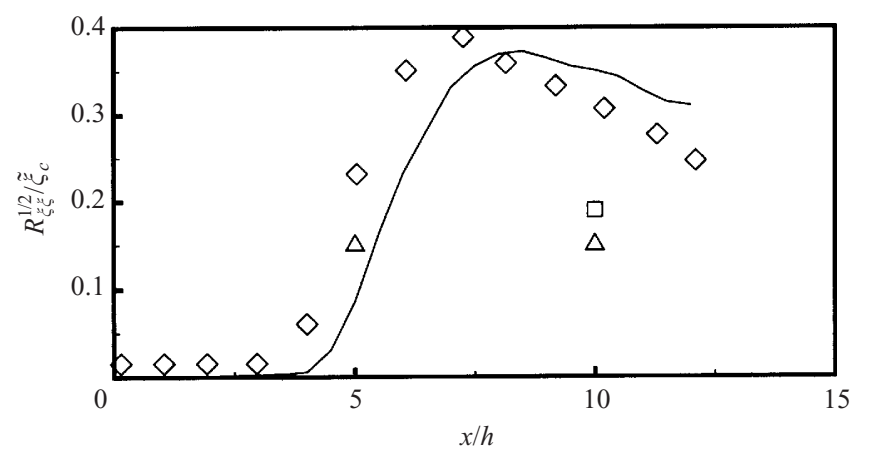

FIGURE 22. Growth of the scalar fluctuation intensity on the jet centreline. - Current results; $\diamond$, Browne et al. (1983); $\square$, Davies et al. (1975); $\triangle$, Ramaprian \& Chandrasekhara (1985).
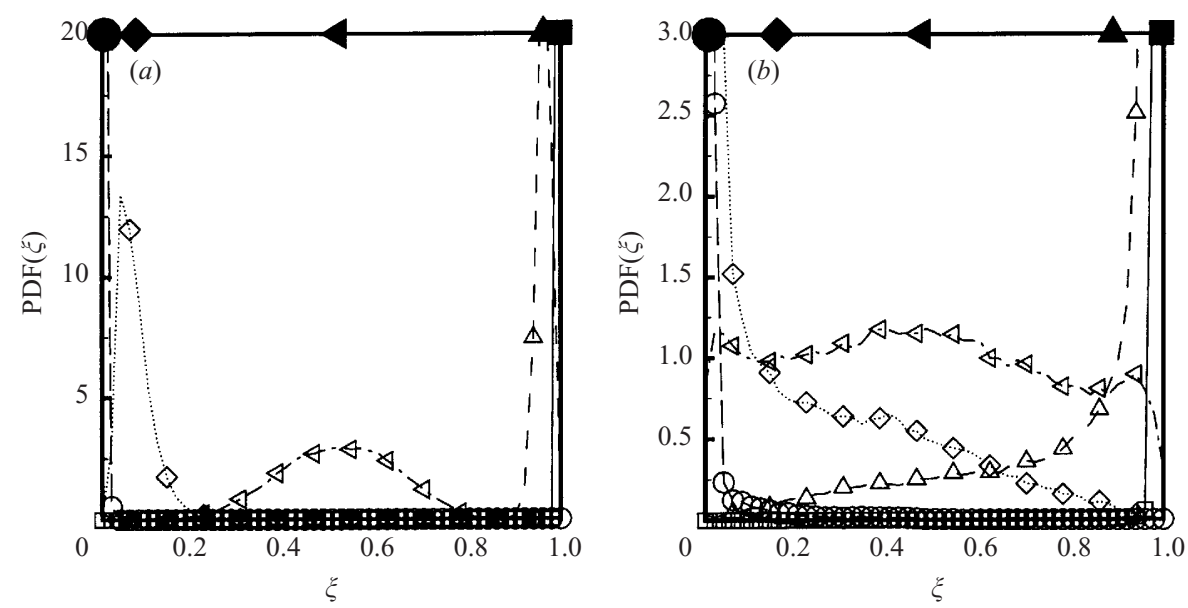

FIGURE 23. Variation of the probability density function of the passive scalar across the jet at (a) $x / h=1.0$ and $\square, y / \delta_{\xi}=0.33 ; \triangle, y / \delta_{\xi}=0.66 ; \triangleleft, y / \delta_{\xi}=1.00 ; \diamond, y / \delta_{\xi}=1.33 ; \bigcirc, y / \delta_{\xi}=1.66$. (b) $x / h=4.0$ and $\square, y / \delta_{\xi}=0 ; \triangle, y / \delta_{\xi}=0.52 ; \triangleleft, y / \delta_{\xi}=1.05 ; \diamond, y / \delta_{\xi}=1.58 ; 0, y / \delta_{\xi}=2.10$.

Figure 22 shows the downstream evolution of the centreline scalar fluctuation intensity, $\boldsymbol{R}_{\xi \xi}$. Consistent with the slow initial development of this jet, the scalar fluctuation intensities evolve slower than experimental ones. The strong growth in the centreline intensities occurs at $x / h=5.0$ for the current jet and is followed by a slow decay downstream, $x / h \geqslant 8.0$. An overshoot in the velocity fluctuations is not observed for this case.

\subsection{Characterization of the mixing process}

The variation of the probability density function of the passive scalar, $\operatorname{PDF}(\xi)$, from the centreline of the jet outwards through the upper shear region is presented here in order to characterize the development of the mixing process in this jet. In all of the PDFs presented, the solid symbols across the top of the plots give the mean scalar value for the PDF with the corresponding symbol. As can be seen in figure 23(a), very near the jet nozzle the mixing process is dominated by the quasi-isotropic velocity fluctuations imposed at the inflow. The variation of the probability density functions across the jet at this station exhibit pure 'marching' behaviour. Note that a 'marching' behaviour refers to the situation where, as the jet is traversed in the cross-stream 

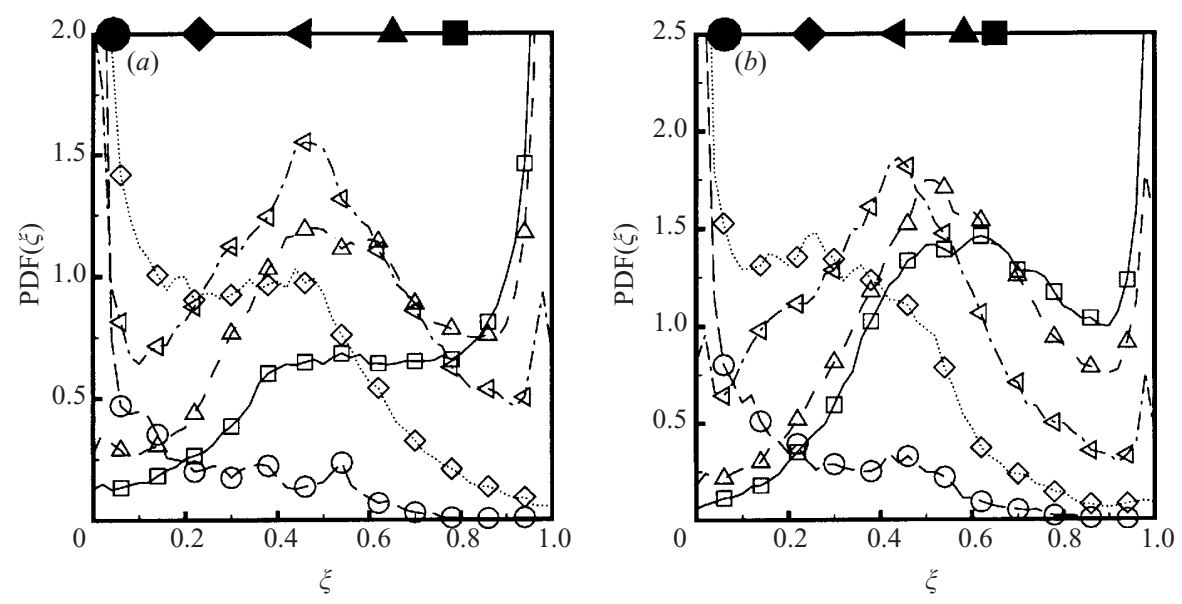

FIGURE 24. Variation of the probability density function of the passive scalar across the jet at (a) $x / h=7.0$ and $\square, y / \delta_{\xi}=0 ; \triangle, y / \delta_{\xi}=0.43 ; \triangleleft, y / \delta_{\xi}=0.87 ; \diamond, y / \delta_{\xi}=1.30 ; 0, y / \delta_{\xi}=1.74$. (b) $x / h=9.0$ and $\square, y / \delta_{\xi}=0 ; \triangle, y / \delta_{\xi}=0.41 ; \triangleleft, y / \delta_{\xi}=0.78 ; \diamond, y / \delta_{\xi}=1.15 ; 0, y / \delta_{\xi}=1.56$.

direction, the peak in each PDF corresponds to the mean scalar value at that lateral location. As would be expected, the PDF at $y / \delta_{\xi}=1.0$ is much wider than the others, indicating stronger mixing by the high-intensity turbulent fluctuations in this region.

Near the end of the potential core at $x / h=4.0$ the probability density functions are very broad, figure $23(b)$. For the lateral location $y / \delta_{\xi}=1.05$, the mean scalar value is $\tilde{\xi}=0.47$; however there is nearly equal probability of having any scalar value in the range $0.05 \leqslant \xi \leqslant 0.9$. For the lateral locations $y / \delta_{\xi}=0.52$ and 1.58 , there is a high probability of having pure jet and pure coflow fluid, respectively, although the PDFs have a central broad region. This streamwise location is in the region where strong vortical structures are beginning to develop, figure 17. It is likely that the broad range of scalar values present at $y / \delta_{\xi}=1.05$ is due to the lateral motion of the region of sharp scalar gradient as vortical structures develop in the velocity field coupled with the occasional strong entrainment of coflow fluid by a structure that develops at $x / h<4.0$.

Figure 24(a) shows the scalar PDFs across the jet at the station $x / h=7.0$. This station is just downstream of the end of the potential core based on the velocity field and is that at which the mean scalar profiles are beginning to show signs of self-similarity. At this station, the probability density functions for $y / \delta_{\xi}=0.43,0.87$ and 1.30 are decidedly non-marching, indicating mixing dominated by large-scale engulfing of fluid. While the mean scalar values, solid shaded symbols at the top of the plot, vary strongly for $0.43 \leqslant y / \delta_{\xi} \leqslant 1.30$, a stationary central peak is present in the PDFs at scalar values $\xi \approx 0.5$. At each of these lateral stations there is a second strong peak in the probability density function corresponding to either pure jet fluid, $y / \delta_{\xi}=0.43$, or pure coflow fluid, $y / \delta_{\xi}=1.30$, or both, $y / \delta_{\xi}=0.87$. For the three locations, the secondary peaks are as strong or stronger than the central peak at $\xi \approx 0.5$, indicating a tendency to have patches of pure jet and pure coflow fluid, interspersed with mixed fluid. The probability density functions for $y / \delta_{\xi}=0$ and 1.74 are quite broad and show a dominant peak of pure jet and pure coflow fluid, respectively. For $y / \delta_{\xi}=0$ the mean scalar value is $\tilde{\xi}=0.79$; however the PDF has a constant value in the region $0.4 \leqslant \xi \leqslant 0.8$.

Figure 24(b) shows the probability density functions of the passive scalar at the 


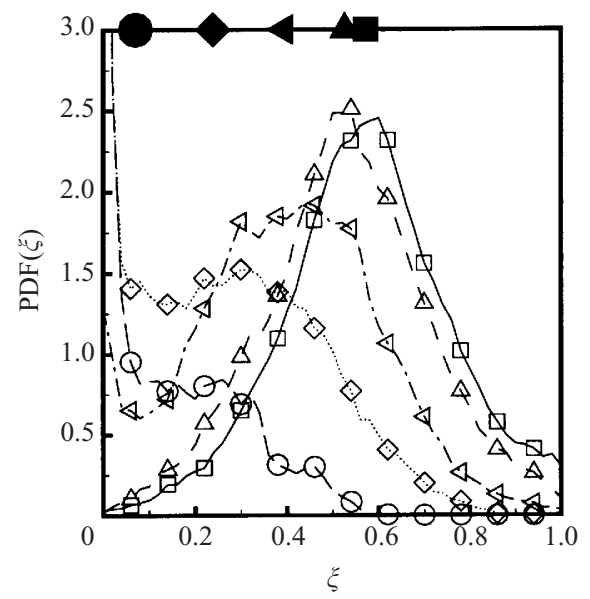

FIGURE 25. Variation of the probability density function of the passive scalar across the jet at $x / h=11.5: \square, y / \delta_{\xi}=0 ; \triangle, y / \delta_{\xi}=0.38 ; \triangleleft, y / \delta_{\xi}=0.72 ; \diamond, y / \delta_{\xi}=1.07 ; \bigcirc, y / \delta_{\xi}=1.45$.

streamwise station $x / h=9.0$ just before the fluctuating scalar and velocity fields reach self-similar behaviour. The mean scalar value at the jet centreline at this station is $\tilde{\xi} \approx 0.65$. However, even at this downstream station there is a high probability of finding pure jet fluid on the centreline. The strong peak corresponding to pure jet fluid in the PDF for $y / \delta_{\xi}=0.41$ is significantly less than that present at $x / h=7.0$ indicating a breakdown of the patches of pure jet fluid away from the centreline due to the effects of small-scale mixing.

The probability density function at $y / \delta_{\xi}=0.78$ still shows the effects of large-scale engulfing of coflow and jet fluid. A strong central peak is present near $\xi=0.42$ with secondary peaks corresponding to pure coflow, $\xi=0$, and pure jet, $\xi=1.0$, fluid. For $y / \delta_{\xi}=1.15$ there is an almost equal probability of finding fluid with $0.1 \leqslant \xi \leqslant 0.5$ as well as a very high probability of pure coflow fluid. The PDFs at this downstream station are of the 'tilted' type (see Karasso \& Mungal 1996) which is evidence of the influence of large-scale engulfment of fluid coupled with small-scale mixing.

Figure 25 shows the probability density functions for the passive scalar field further downstream in the jet, $x / h=11.5$, after the fluctuating velocity and scalar fields have obtained self-similar profiles. By this station, small-scale mixing has eliminated the presence of strong patches of unmixed jet fluid even at the jet centreline. The probability density functions at $y / \delta_{\xi}=0$ and 0.38 exhibit 'marching' type behaviour at this streamwise station, having a single peak which corresponds to the local mean value of the passive scalar. At the lateral locations $y / \delta_{\xi}=0.72$ and 1.07 the probability density functions of the passive scalar still show a central peak which tends to follow the local mean scalar value. However, at the stations in the outer half of the jet, closer to the coflow fluid, there are strong peaks in the PDFs corresponding to pure coflow fluid.

\section{Conclusions}

A computational model has been developed for a three-dimensional, spatially evolving, turbulent plane jet using direct numerical simulation of the compressible Navier-Stokes equations. This computational model utilizes accurate numerical techniques to deal with the complex issues in unsteady flow computations arising due 
to the open computational domain. The efficiency and accuracy, both temporal and spatial, of this computational model is suitable for performing detailed studies of spatially evolving turbulent flows. This simulation provides detailed data on all flowfield variables in the initial and later, approximately self-similar, regions of spatially evolving turbulent shear flows which, being validated against laboratory experiments, can be utilized in the evaluation and formulation of subgrid models for large-eddy simulation.

The Reynolds number at the nozzle is $R e_{h}=3000$, which increases to $R e=4838$ based on the jet half-width. The self-similar jet growth rate, $K_{1 u}=0.092$, compares well with the values $K_{1 u}=0.110$ and $K_{1 u}=0.100$ observed experimentally by Ramaprian \& Chandrasekhara (1985) and Gutmark \& Wygnanski (1976) respectively. Likewise, the self-similar centreline velocity decay rate, $C_{1 u}=0.201$, agrees with the values $C_{1 u}=0.220$ measured by Thomas \& Prakash (1991) and $C_{1 u}=0.189$ measured by Gutmark \& Wygnanski (1976).

The mean longitudinal velocity profiles are self-similar at $x / h=7.0$ and Reynolds stress profiles are approximately self-similar by $x / h=10.0$. The mean and Reynolds stress profiles show good agreement with the experimental data of Ramaprian \& Chandrasekhara (1985) and Gutmark \& Wygnanski (1976). The streamwise growth of the fluctuating velocity components is in good agreement with experimental data, although there are some differences in the initial growth rate of the centreline rootmean-square longitudinal velocity as well as the lateral component. This, however, is not surprising since the downstream development of planar jets is sensitive to nozzle and external conditions. While the shear-layer momentum thickness of the current results is somewhat larger than experimental values, the inflow fluctuation intensity and broadband spectrum have been designed to provide a rapid breakdown of the jet to a fully developed turbulent state.

As shown through the use of coherency spectra across the jet, the simulation captures well the strong growth in the shear layer mode near the jet nozzle. A shift of the dominant frequency in the coherency spectra is observed over the length of the potential core from the shear layer mode near the inflow to that corresponding to the jet column mode. The transition to the dominance of the jet column mode occurs at the end of the potential core and thus coincides with the emergence of typical jet-like mean longitudinal velocity profiles. The region in which the jet column mode becomes dominant, $3.0 \leqslant x / h \leqslant 5.0$, corresponds to that over which the strong breakdown to three-dimensionality occurs in the jet. Over this range, there is a substantial decrease of the longitudinal and spanwise correlation scales as well as a large increase in the energy content of the intermediate-to-small length scales in the turbulence.

The turbulent-kinetic-energy balance in the self-similar region from the DNS simulation generally compares well with those of Ramaprian \& Chandrasekhara (1985) and Gutmark \& Wygnanski (1976). Strong production is observed in the region of sharp mean gradient with turbulent transport towards the jet centreline and edges. The viscous dissipation is relatively uniform across the core of the jet, $-1 \leqslant y / \delta_{U} \leqslant 1$. The present results, however, have the advantage of allowing direct calculation of all terms in the TKE balance. It is shown that on the centreline of the jet the small scales of motion are substantially more isotropic than at the jet edges, and that the isotropic approximation for the dissipation results in an error lower than $3 \%$.

Comparisons have been made of the evolution of a passive scalar field in a planar turbulent jet to experimental data on the temperature field in heated planar jets. Mean scalar profiles compare well with mean temperature profiles from experimental studies. The initial evolution of the centreline scalar decay rate and jet width based 
on the passive scalar are somewhat slower in the current results due to differences between the inflow conditions in the DNS and experiments. However, the downstream growth rates and centreline decay rates compare well with experimental values.

Analysis of the probability density functions for the passive scalar has been used to characterize the evolution of the mixing process in turbulent planar jets. Near the nozzle, prior to vortex rollup, the probability density functions are dominated by the effects of the quasi-isotropic small-scale fluctuations imposed at the inflow. Therefore, in this region, scalar PDFs exhibit classical marching behaviour. Downstream, after the rollup of strong vortical structures in the shear layers on either side of the potential core, the mixing process is dominated by large-scale engulfing of coflow fluid into the jet by these structures. In this region after vortex rollup, the probability density functions across the jet are non-marching.

However, when the flow field in the jet becomes more fully turbulent downstream, the influence of large-scale engulfing of fluid on the mixing near the jet centreline progressively decreases. In the core of the self-similar region of the jet, the mixing process is dominated by small-scale mixing. Therefore, the probability density functions of the passive scalar are of the marching type. However, near the jet edges, the engulfing of coflow fluid by the presence of large structures remains significant. Therefore, double peaks are present in the PDFs, one of which corresponds to pure fluid originating in the coflow.

In summary, the present work demonstrates, through detailed comparison with classical experimental data, that DNS with high-order space and time accuracy and appropriate schemes to handle inflow and outflow boundaries can successfully represent a spatially evolving plane jet. The development from interacting shear layers near the nozzle to the self-similar jet can be captured, albeit at a moderate Reynolds number. Such validated DNS can be useful in developing and validating improved turbulence prediction methods as well as in refining our knowledge of mixing in turbulent jets.

Support for the first author was provided by the Applied Mathematical Sciences Program of the DOE Office of Mathematics, Information, and Computational Sciences under contract DE-AC03-76SF00098 as well as by the Department of Energy Computational Sciences Graduate Fellowship Program. Partial support for the second and third authors was provided by AFOSR through grant F49620-96-1-0106 and ONR through grant N00014-99-1-0745. This work was supported in part by a grant of HPC time from the Naval Oceanographic Office Department of Defense Major Shared Resource Center.

\section{REFERENCES}

Albertson, M. L., DAi, Y. B., Jenson, R. A. \& House, H. 1950 Diffusion of submerged jets. Trans. ASME 115, 639-664.

BATT, R. G. 1977 Turbulent mixing of passive and chemically reacting species in a low-speed shear layer. J. Fluid Mech. 82, 53-95.

Bell, J. H. \& Mehta, R. D. 1990 Development of a two-stream mixing layer from tripped and untripped boundary layers. AIAA J. 28, 2034-2042.

Bradbury, L. J. S. 1965 The structure of a self-preserving turbulent plane jet. J. Fluid Mech. 23, $31-64$.

Bradbury, L. J. S. \& Riley, J. 1967 The spread of a turbulent jet issuing into a parallel moving airstream. J. Fluid Mech. 27, 381-394.

Browne, L. W. B., Antonia, R. A., Rajagopalan, S. \& Chambers, A. J. 1983 Interaction region of a 
two-dimensional turbulent plane jet in still air. In Structure of Complex Turbulent Shear Flow, IUTAM Symposium, Marseille 1982 (ed. R. Dumas \& L. Fulachier), pp. 411-419. Springer.

Carpenter, M. H., Gottlieb, D. \& Abarbanel, S. 1993 The stability of numerical boundary treatments for compact high-order finite-difference schemes. J. Comput. Phys. 108, 272-295.

Carpenter, M. H. \& Kennedy, C. A. 1994 Fourth-order 2n-storage Runge-Kutta schemes. NASA Tech. Mem. 109112.

Comte, P., Lesieur, M., Laroche, H. \& Normand, X. 1989 Numerical simulations of turbulent plane shear layers. In Turbulent Shear Flows 6 (ed. J.-C. André, J. Cousteix, F. Durst, B. E. Launder, F. W. Schmidt \& J. H. Whitelaw), pp. 361-380. Springer.

Dahm, W. J. A. \& Dimotakis, P. E. 1990 Mixing at large Schmidt number in the self-similar far field of turbulent jets. J. Fluid Mech. 217, 299-330.

Dai, Y., Kobayashi, T. \& Taniguchi, N. 1994 Large eddy simulation of plane turbulent jet flow using a new outflow velocity boundary condition. JSME Intl J. B Fluids and Thermal Engng 37, 242-253.

Davies, A. E., Keffer, J. F. \& Baines, W. D. 1975 Spread of a heated plane turbulent jet. Phys. Fluids 18, 770-775.

Everitt, K. W. \& Robins, A. G. 1978 The development and structure of turbulent plane jets. J. Fluid Mech. 88, 563-583.

GeORGE, W. K. 1989 The self-preservation of turbulent flows and its relation to initial conditions and coherent structures. In Advances in Turbulence (ed. W. K. George \& R. Arndt), pp. 39-73. Hemisphere.

Gutmark, E. \& Wygnanski, I. 1976 The planar turbulent jet. J. Fluid Mech. 73, 465-495.

Heskestad, G. 1965 Hot-wire measurements in a plane turbulent jet. Trans. ASME: J. Appl. Mech. 32, 721-734.

Hu, F. Q. 1996 On absorbing boundary conditions for linearized Euler equations by a perfectly matched layer. J. Comput. Physics 129, 201-219.

Hussain, A. K. M. F. \& Clark, A. R. 1977 Upstream influence on the near field of a plane turbulent jet. Phys. Fluids 20, 1416-1426.

Jenkins, P. E. \& Goldschmidt, V. W. 1973 Mean temperature and velocity in a plane turbulent jet. Trans. ASME: J. Fluids Engng 95, 581-584.

Karasso, P. S. \& Mungal, M. G. 1996 Scalar mixing and reaction in plane liquid shear layers. J. Fluid Mech. 323, 23-63.

Koochesfahani, M. M. \& Dimotakis, P. E. 1986 Mixing and chemical reactions in a turbulent liquid mixing layer. J. Fluid Mech. 170, 83-112.

LaRue, J. C., Ly, T., Rahai, H. \& Jan, P. Y. 1997 On similarity of a plane turbulent jet in a co-flowing stream. In Proc. Eleventh Symp. on Turbulent Shear Flows, vol. 3, pp. 25.11-25.16. Springer.

Lasheras, J. C. \& Chol, H. 1988 Three-dimensional instability of a plane free shear layer: An experimental study of the formation and evolution of streamwise vortices. J. Fluid Mech. 189, 53-86.

Le Ribault, C., Sarkar, S. \& Stanley, S. A. 1999 Large eddy simulation of a plane jet. Phys. Fluids 11, 3069-3083.

Le Ribault, C., Sarkar, S. \& Stanley, S. A. 2001 Large eddy simulation of the evolution of a passive scalar in a plane jet. AIAA J. 39, 1509-1516.

LELE, S. K. 1992 Compact finite difference schemes with spectral-like resolution. J. Comput. Phys. 103, 16-42.

Michalke, A. 1965 On spatially growing disturbances in an inviscid shear layer. J. Fluid Mech. 23, 521-544.

Miller, D. R. \& Comings, E. W. 1957 Static pressure distributions in the free turbulent jet. J. Fluid Mech. 3, 1-16.

Monkewitz, P. A. \& Huerre, P. 1982 Influence of the velocity ratio on the spatial instability of mixing. Phys. Fluids 25, 1137-1143.

Moser, R. D., Rogers, M. M. \& Ewing, D. W. 1998 Self-similarity of time-evolving plane wakes. J. Fluid Mech. 367, 255-289.

Poinsot, T. J. \& Lele, S. K. 1992 Boundary conditions for direct simulations of compressible viscous flows. J. Comput. Phys. 101, 104-129. 
Ramaprian, B. R. \& Chandrasekhara, M. S. 1985 LDA measurements in plane turbulent jets. Trans. ASME: J. Fluids Engng 107, 264-271.

Reichert, R. S. \& Biringen, S. 1997 Numerical simulation of compressible plane jets. AIAA Paper 97-1924.

Rogers, M. M. \& Moser, R. D. 1994 Direct simulation of a self-similar turbulent mixing layer. Phys. Fluids 6, 903-923.

Rudy, D. H. \& StRIKwERDA, J. C. 1980 A nonreflecting outflow boundary condition for subsonic Navier-Stokes calculations. J. Comput. Phys. 36, 55-70.

SPENCER, B. W. \& Jones, B. G. 1971 Statistical investigation of pressure and velocity fields in the turbulent two-stream mixing layer. AIAA Paper 71-613.

Stanley, S. \& SARKar, S. 1997 a Simulations of spatially developing plane jets. AIAA Paper 97-1922.

StANLEY, S. \& SARKAR, S. $1997 b$ Simulations of spatially developing two-dimensional shear layers and jets. Theor. Comput. Fluid Dyn. 9, 121-147.

Stanley, S. A. \& Sarkar, S. 2000 Influence of nozzle conditions and discrete forcing on turbulent planar jets. AIAA J. 38, 1615-1623.

Tennekes, H. \& Lumley, J. L. 1972 A First Course in Turbulence. The MIT Press.

Thomas, F. O. \& CHU, H. C. 1989 An experimental investigation of the transition of a planar jet: Subharmonic suppression and upstream feedback. Phys. Fluids A 1, 1566-1587.

Thomas, F. O. \& Prakash, K. M. K. 1991 An experimental investigation of the natural transition of an untuned planar jet. Phys. Fluids A 3, 90-105.

Thompson, K. W. 1987 Time dependent boundary conditions for hyperbolic systems. J. Comput. Phys. 68, 1-24.

Thompson, K. W. 1990 Time-dependent boundary conditions for hyperbolic systems ii. J. Comput. Phys. 89, 439-461.

Weinberger, C., Rewerts, J. \& Janicka, J. 1997 The influence of inlet conditions on a large eddy simulation of a turbulent plane jet. In Proc. Eleventh Symp. on Turbulent Shear Flows, vol. 3, pp. 25.17-25.22. Springer.

Williams, F. A. 1985 Combustion Theory, 2nd edn. Addison-Wesley.

Wygnanski, I. \& Fiedler, H. E. 1970 The two-dimensional mixing region. J. Fluid Mech. 41, $327-361$. 